\title{
DEVELOPMENT OF A DISPERSION RELATION-PRESERVING UPWINDING SCHEME FOR INCOMPRESSIBLE NAVIER-STOKES EQUATIONS ON NONSTAGGERED GRIDS
}

\author{
P. H. Chiu, Tony W. H. Sheu, and R. K. Lin \\ Department of Engineering Science and Ocean Engineering, National Taiwan \\ University, Taipei, Taiwan, Republic of China
}

In this article a scheme which preserves the dispersion relation for convective terms is proposed for solving the two-dimensional incompressible Navier-Stokes equations on nonstaggered grids. For the sake of computational efficiency, the splitting methods of Adams-Bashforth and Adams-Moulton are employed in the predictor and corrector steps, respectively, to render second-order temporal accuracy. For the sake of convective stability and dispersive accuracy, the linearized convective terms present in the predictor and corrector steps at different time steps are approximated by a dispersion relation-preserving (DRP) scheme. The DRP upwinding scheme developed within the 13-point stencil framework is rigorously studied by virtue of dispersion and Fourier stability analyses. To validate the proposed method, we investigate several problems that are amenable to exact solutions. Results with good rates of convergence are obtained for both scalar and Navier-Stokes problems.

\section{INTRODUCTION}

When solving the two-dimensional convection-diffusion equation, the dispersion error, which is defined as the difference between the effective and actual wave numbers, cannot be completely eliminated. A reliable scheme for solving the practically and academically important convection-dominated convection-diffusion transport equation and Navier-Stokes equations at high Reynolds numbers must have the ability to avoid convective instability. To overcome the convective instability, many upwind schemes have been proposed; see, e.g., [1-5]. This author has also proposed monotonic upwind schemes by virtue of the underlying idea of an M-matrix to enhance convective stability [6,7]. One theory that may be adopted to enhance convective stability is to take the dispersive nature of the investigated first derivative term into consideration [8].

The scheme used for approximation of the convection term can preserve the dispersion relation if it accommodates the same dispersion relation as that of the original first-order partial derivative term [8]. This relation, which is derived by

Received 3 June 2005; accepted 15 July 2005.

Address correspondence to Tony W. H. Sheu, Department of Engineering Science and Ocean Engineering, National Taiwan University, No. 1, Sec. 4, Roosevelt Road, Taipei, Taiwan, Republic of China. E-mail: twhsheu@ntu.edu.tw 


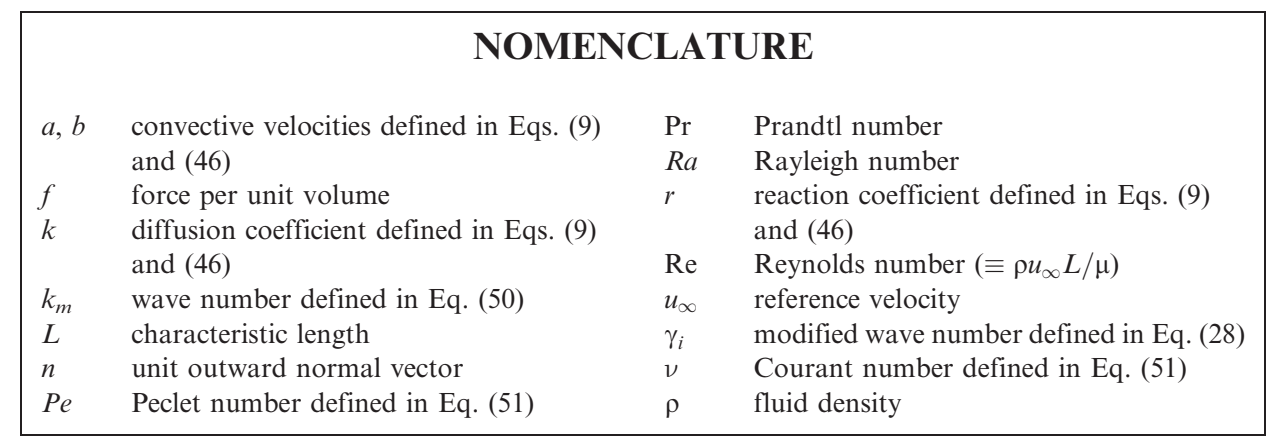

taking the spatial Fourier transform of the first-derivative term, reveals how the angular frequency relates to the wave number of the spatial variable [9]. The main reason for employing a dispersion relation-preserving (DRP) scheme is that the resulting dispersiveness, dissipation, and group and phase velocities of each wave component supported by the first-order derivative can be well modeled [10]. In the above light, we conduct in this article a standard modified equation analysis, which involves truncated Taylor series, together with a Fourier transform analysis [11], which enables us to derive the same or almost the same dispersion relation as the original partial differential equation, in the approximation of convective terms.

This article is organized as follows. Section 2 presents the working NavierStokes equations in primitive-variable form. This is followed by presentation of the rationale for advocating the segregated solution algorithm on nonstaggered grids. Section 4 presents a splitting method for the sake of computational efficiency, and Section 5 describes the two-dimensional DRP scheme for the approximation of first-order derivative terms. Section 6 addresses the dispersion and Fourier (or von Neumann) stability analyses of the proposed CDR discretization scheme. Section 7 presents simulated results, by which the proposed DRP upwinding method is validated. In Section 8 we give concluding remarks.

\section{WORKING EQUATIONS}

In this study we investigate the incompressible viscous fluid flow, which is governed by the continuity and Navier-Stokes equations for the chosen pair of primitive variables $(\underline{u}, p)$ :

$$
\begin{aligned}
\nabla \cdot \underline{u} & =0 \\
\frac{\partial \underline{u}}{\partial t}+\underline{u} \cdot \nabla \underline{u} & =-\nabla p+\frac{1}{\operatorname{Re}} \nabla^{2} \underline{u}+\underline{f}
\end{aligned}
$$

Given an initial divergence-free velocity field, the velocity vector $\underline{u}$ and pressure $p$ are sought subject to the boundary velocity. The length is scaled by $L$, the velocity components by $u_{\infty}$, the time by $L / u_{\infty}$, and the pressure by $\rho u_{\infty}^{2}$, where $\rho$ denotes the constant fluid density. The Reynolds number Re shown in Eq. (2) appears as a consequence of the above normalization. 
Momentum equations can be solved together with the constraint equation (1). With this unconditional fluid incompressibility, the resulting matrix system may be ill-conditioned. Under the circumstances, convergent solutions for $(\underline{u}, p)$ become very difficult to obtain using the computationally less expensive iterative solvers [12]. The peripheral storage for the matrix equations may exceed our available computer power and disk space. These drawbacks prompted the use of the pressure Poisson equation (PPE) approach [13] to eliminate the difficulty encountered in the mixed formulation. Within the segregated analysis framework, equation for $p$ is, thus, derived to replace Eq. (1). By applying a divergence operator on Eq. (2), we are led to derive the following Poisson equation:

$$
\nabla^{2} p=\nabla\left(-\frac{\partial \underline{u}}{\partial t}+\frac{1}{\operatorname{Re}} \nabla^{2} \underline{u}-\underline{u} \cdot \nabla \underline{u}+\underline{f}\right)
$$

The above PPE approach is subject to theoretically rigorous integral boundary condition [14]. Equation (2) is, therefore, computationally more challenging to solve. For this reason, we adopt in this study the conventional differential-type boundary condition given below:

$$
\frac{\partial p}{\partial n}=\left(-\frac{\partial \underline{u}}{\partial t}+\frac{1}{\operatorname{Re}} \nabla^{2} \underline{u}-\underline{u} \cdot \nabla \underline{u}+\underline{f}\right) \cdot \underline{n}
$$

In the above, $\underline{n}$ denotes the unit outward normal vector to the domain boundary.

\section{DISCRETIZATION OF INCOMPRESSIBLE NAVIER-STOKES EQUATIONS ON NONSTAGGERED GRIDS}

Use of staggered grid approaches, which have been successfully applied to suppress oscillations arising from even-odd coupling, increases the coding complexity and may consume more computational time. This provided the motivation for discretizing the partial differential equations over a domain in which both the velocities and pressure are stored at the same point. In the nonstaggered mesh, care should be taken for $\nabla p$ order to avoid spurious oscillations in the pressure field.

Our idea behind avoiding even-odd decoupling solutions is to take the nodal value of $p_{j}$ into account when approximating $\nabla p$ at an interior node $j$ [15]. Rather than attempting to explicitly approximate $p_{x}$ at node $j$, the value of $\left.p_{x}\right|_{j}$ is obtained implicitly along with two adjacent values $\left.p_{x}\right|_{j \pm 1}$. Define $F_{j}$ as

$$
F_{j}=\left.h p_{x}\right|_{j}
$$

where $h$ denotes the constant mesh size. The method adopted in the present study for solving the nodal value of $F$ is the following implicit equation:

$$
\begin{aligned}
c_{1} F_{j+1}+c_{2} F_{j}+c_{3} F_{j-1}= & c_{4}\left(p_{j+2}-p_{j+1}\right)+c_{5}\left(p_{j+1}-p_{j}\right) \\
& +c_{6}\left(p_{j}-p_{j-1}\right)+c_{7}\left(p_{j-1}-p_{j-2}\right)
\end{aligned}
$$

The seven undetermined coefficients are determined by expanding $F_{j \pm 1}$ in Taylor series with respect to $F_{j}$, and $p_{j \pm 1}$ and $p_{j \pm 2}$ with respect to $p_{j}$. By substituting these 
expansions into Eq. (6) and using Eq. (5), a simultaneous set of algebraic equations can be obtained for uniquely determining $c_{1}-c_{7}$. On physical grounds, it is legitimate to set $c_{1}=c_{3}$ since $p$ is elliptic in nature owing to the Poisson equation given in (3). Having set $c_{1}=c_{3}$, other coefficients can be determined as $c_{1}=1 / 5, c_{2}=3 / 5$, $c_{4}=1 / 60, c_{5}=29 / 60, c_{6}=29 / 60$, and $c_{7}=1 / 60$. As for the derivation of working equation for $F$ at a node immediately adjacent to the right boundary point (for example), we can employ Eq. (6) at the limiting condition of $c_{1}=c_{4}=c_{5}=0$. With the expression for boundary $F$ via Eq. (6), interior values of $F_{j}\left(2 \leq j \leq j_{\max }-1\right)$ can be obtained with less computational expense from the banded tridiagonal matrix equations.

\section{SPLITTING SCHEME FOR NAVIER-STOKES EQUATIONS}

When solving the momentum equation (2) for velocity components and the Poisson equation (3) for pressure, considerable iterations are needed to reach convergence for equation nonlinearity. To avoid excessive computing time, the predictorcorrector splitting scheme will be employed together with explicit treatment of the pressure term [16]. In the predictor step, the predictor velocity is solved using the second-order-accurate Adams-Bashforth scheme. In the corrector step, the momentum equation is then solved using the second-order-accurate Adams-Moulton scheme:

Explicit predictor step:

$$
\begin{aligned}
& \frac{\underline{\underline{u}}^{n+1}-\underline{u}^{n}}{\Delta t}+\left[\frac{3}{2}(\underline{u} \cdot \nabla \underline{u})^{n}-\frac{1}{2}(\underline{u} \cdot \nabla \underline{u})^{n-1}\right] \\
& =+\left[\frac{3}{2}(-\nabla p)^{n}-\frac{1}{2}(-\nabla p)^{n-1}\right]+\left[\frac{3}{2}\left(\frac{1}{\operatorname{Re}} \nabla^{2} \underline{u}\right)^{n}-\frac{1}{2}\left(\frac{1}{\operatorname{Re}} \nabla^{2} \underline{u}\right)^{n-1}\right]+\underline{f}^{n+1 / 2}
\end{aligned}
$$

Implicit corrector step:

$$
\begin{aligned}
& \frac{\underline{u}^{n+1}-\underline{u}^{n}}{\Delta t}+\frac{1}{2}\left[\left(\underline{\tilde{u}}^{n+1} \cdot \nabla \underline{u}^{n+1}\right)+\left(\underline{u}^{n} \cdot \nabla \underline{u}^{n}\right)\right] \\
& =+\left[\frac{3}{2}(-\nabla p)^{n}-\frac{1}{2}(-\nabla p)^{n-1}\right]+\frac{1}{2}\left[\left(\frac{1}{\operatorname{Re}} \nabla^{2} \underline{u}\right)^{n+1}+\left(\frac{1}{\operatorname{Re}} \nabla^{2} \underline{u}\right)^{n}\right]+f^{n+1 / 2}
\end{aligned}
$$

This is followed by solving the Poisson equation for pressure (3) using the updated velocity to complete the calculation within one time interval. Note that the above splitting algorithm involves only two convection-diffusion-reaction (CDR) scalar equations in the corrector step and one Poisson equation. No iteration is needed.

\section{DISPERSION RELATION-PRESERVING CONVECTION SCHEME}

Considering the following constant coefficient convection-diffusion-reaction model equation, which is the semidiscretized equation for $\phi_{t}+a \phi_{x}+b \phi_{y}-k \nabla^{2} \phi=f$ : 


$$
\frac{\phi^{n+1}-\phi^{n}}{\Delta t}+a \phi_{x}+b \phi_{y}-k \nabla^{2} \phi=f
$$

Note that the above equation bears a close similarity to Eq. (8). In this study, we employ the standard second-order central difference for the diffusion term. In the grid system schematic in Figure 1, the first-order spatial derivative terms shown in (9) are approximated as follows in the case of $\Delta x=\Delta y=h$. Taking $\phi_{x}$ as an example, it can be approximated as follows:

$$
\begin{aligned}
\phi_{x}(x, y) \simeq \frac{1}{h} & \left(a_{1} \phi_{i-1, j-1}+a_{2} \phi_{i, j-1}+a_{3} \phi_{i+1, j-1}+a_{4} \phi_{i-1, j}\right. \\
& +a_{5} \phi_{i, j}+a_{6} \phi_{i+1, j}+a_{7} \phi_{i-1, j+1}+a_{8} \phi_{i, j+1}+a_{9} \phi_{i+1, j+1} \\
& \left.+a_{10} \phi_{i, j-2}+a_{11} \phi_{i, j+2}+a_{12} \phi_{i-2, j}\right) \quad a>0
\end{aligned}
$$

and

$$
\begin{aligned}
\phi_{x}(x, y) \simeq \frac{1}{h}( & a_{1} \phi_{i-1, j-1}+a_{2} \phi_{i, j-1}+a_{3} \phi_{i+1, j-1}+a_{4} \phi_{i-1, j} \\
& +a_{5} \phi_{i, j}+a_{6} \phi_{i+1, j}+a_{7} \phi_{i-1, j+1}+a_{8} \phi_{i, j+1}+a_{9} \phi_{i+1, j+1} \\
& \left.+a_{10} \phi_{i, j-2}+a_{11} \phi_{i, j+2}+a_{13} \phi_{i+2, j}\right) \quad a<0
\end{aligned}
$$

In what follows, the scheme will be presented for the case with $a>0$. By applying the Taylor series expansions for $\phi_{i \pm 1, j}, \phi_{i-2, j}, \phi_{i, j \pm 1}, \phi_{i, j \pm 2}, \phi_{i \pm 1, j \pm 1}$, the leading 11

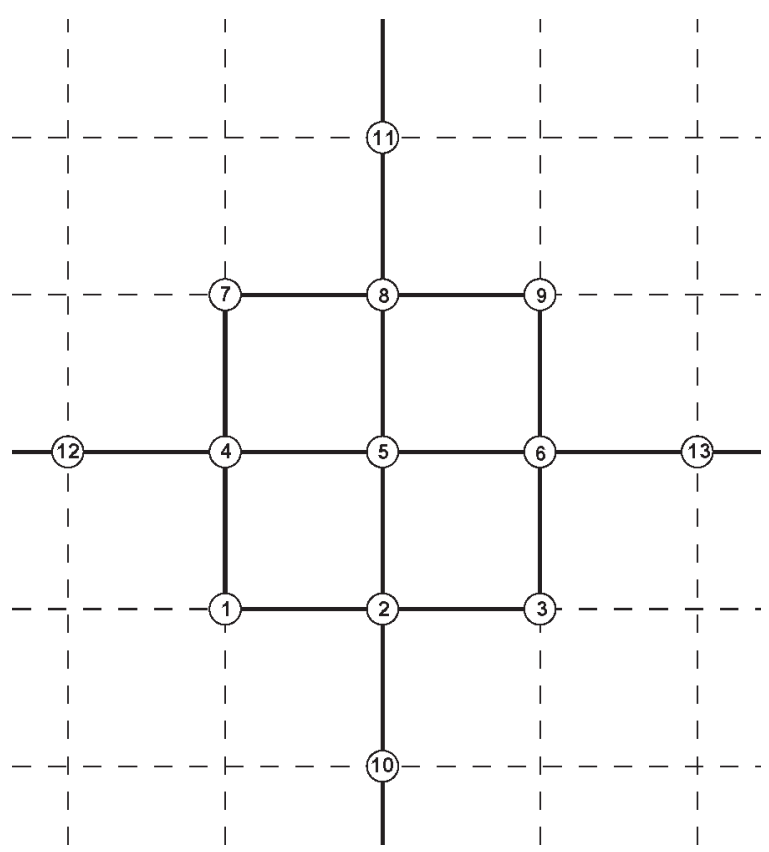

Figure 1. Schematic of the 13 stencil points. 
error terms $\phi, \phi_{x}, \phi_{y}, \phi_{x x}, \phi_{x y}, \phi_{y y}, \phi_{x x y}, \phi_{x y y}, \phi_{x x x}, \phi_{y y y}$, and $\phi_{x x y y}$ shown in the resulting modified equation will be eliminated to derive the following system of algebraic equations:

$$
\begin{aligned}
a_{1}+a_{2}+a_{3}+a_{4}+a_{5}+a_{6}+a_{7}+a_{8}+a_{9}+a_{10}+a_{11}+a_{12} & =0 \\
-a_{1}+a_{3}-a_{4}+a_{6}-a_{7}+a_{9}-2 a_{12} & =1 \\
-a_{1}-a_{2}-a_{3}+a_{7}+a_{8}+a_{9}-2 a_{10}+2 a_{11} & =0 \\
a_{1}+a_{3}+a_{4}+a_{6}+a_{7}+a_{9}+4 a_{12} & =0 \\
a_{1}-a_{3}-a_{7}+a_{9} & =0 \\
a_{1}+a_{2}+a_{3}+a_{7}+a_{8}+a_{9}+4 a_{10}+4 a_{11} & =0 \\
-a_{1}-a_{3}+a_{7}+a_{9} & =0 \\
-a_{1}+a_{3}-a_{7}+a_{9} & =0 \\
-a_{1}+a_{3}-a_{4}+a_{6}-a_{7}+a_{9}-8 a_{12} & =0 \\
-a_{1}-a_{2}-a+3+a_{7}+a_{8}+a_{9}-8 a_{10}+8 a_{11} & =0 \\
a_{1}+a_{3}+a_{7}+a_{9} & =0
\end{aligned}
$$

One more equation is needed for uniquely determining the coefficients $a_{1}-a_{12}$ shown in (10).

As convection highly dominates diffusion, approximation of $\phi_{x}$ to retain the dispersion relation, which signifies the relation between the angular frequency of the wave and the wave number of the spatial variable, is essential for effective suppression of the possible convective instability in the course of approximating $\phi_{x}$ [17]. It is, thus, desirable that the right-hand side of (10) is made to have nearly the same Fourier transform in space as the original partial derivative shown in the left-hand side of (10). Within the DRP analysis framework [8, 18], which has been applied with great success to approximate $\phi_{x}$ in the one-dimensional context, define first the Fourier transform and its inverse for $\phi(x, y)$ in two space dimensions as follows:

$$
\begin{gathered}
\widetilde{\phi}(\alpha, \beta)=\frac{1}{(2 \pi)^{2}} \int_{-\infty}^{+\infty} \int_{-\infty}^{+\infty} \phi(x, y) e^{-\mathbf{i}(\alpha x+\beta y)} d x d y \\
\phi(x, y)=\int_{-\infty}^{+\infty} \int_{-\infty}^{+\infty} \widetilde{\phi}(\alpha, \beta) e^{\mathbf{i}(\alpha x+\beta y)} d \alpha d \beta
\end{gathered}
$$

By conducting Fourier transform on terms shown in both sides of (10), we are led to derive the first component of the actual wave-number vector $\underline{\alpha}=(\alpha, \beta)$ :

$$
\begin{aligned}
\alpha \simeq \frac{-\mathbf{i}}{h} & \left(a_{1} e^{-\mathbf{i}(\alpha h+\beta h)}+a_{2} e^{-\mathbf{i} \beta h}+a_{3} e^{\mathbf{i}(\alpha h-\beta h)}+a_{4} e^{-\mathbf{i} \alpha h}\right. \\
& +a_{5}+a_{6} e^{\mathbf{i} \alpha h}+a_{7} e^{-\mathbf{i}(\alpha h-\beta h)}+a_{8} e^{\mathbf{i} \beta h}+a_{9} e^{\mathbf{i}(\alpha h+\beta h)} \\
& \left.+a_{10} e^{\mathbf{i}(-\beta h)}+a_{11} e^{\mathbf{i}(\beta h)}+a_{12} e^{\mathbf{i}(-\alpha h)}\right)
\end{aligned}
$$

In approximation sense, the effective wave number $\widetilde{\alpha}$ in $\underline{\widetilde{\alpha}}=(\widetilde{\alpha}, \widetilde{\beta})$ can be regarded as the right-hand side of (25) [8]: 

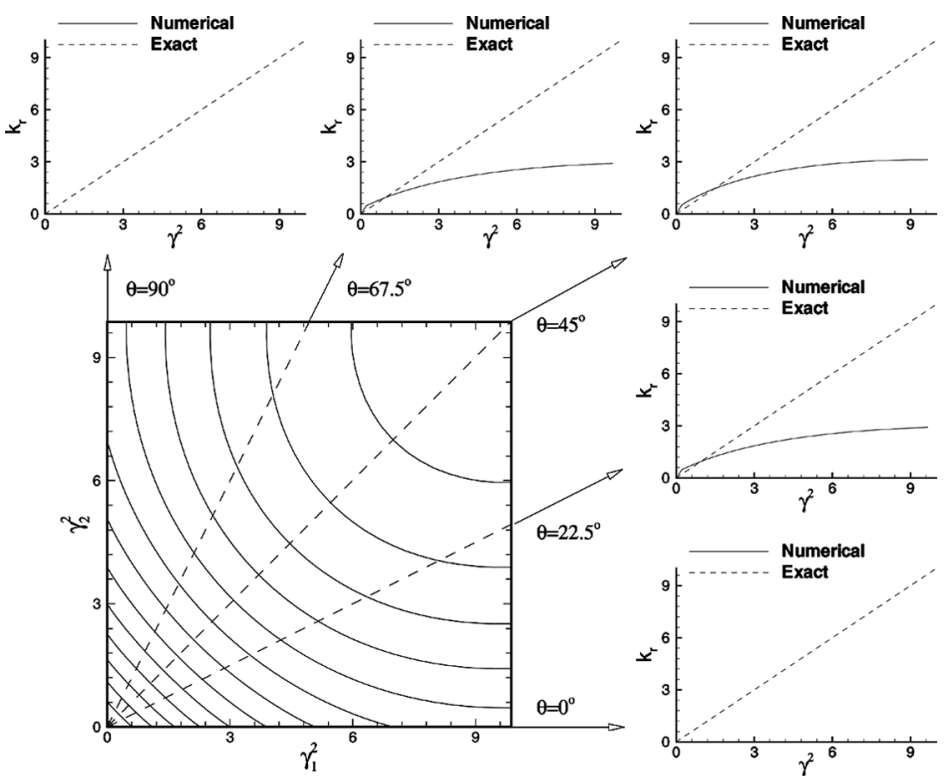

(a)
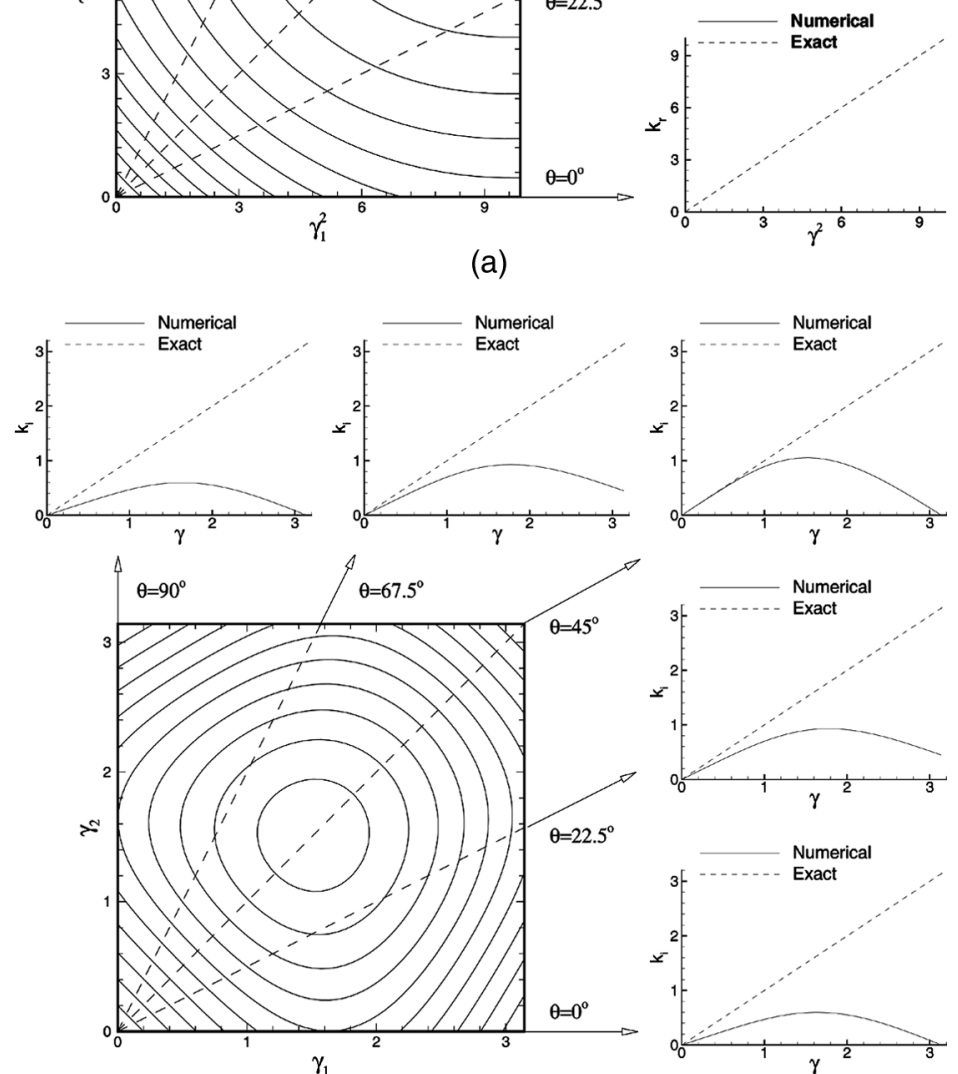

(b)

Figure 2. Plots of $k_{r}$ and $k_{i}$ against $\left(\gamma_{1}^{2}, \gamma_{2}^{2}\right)$ and $\left(\gamma_{1}, \gamma_{2}\right)$, respectively, for the proposed scheme considered at $\mathrm{Pe}_{x}=\mathrm{Pe}_{y}=10^{3}, R_{x}=R_{y}=0$, and $\nu_{x}=\nu_{y}=0.2:(a) k_{r} ;(b) k_{i}$. 

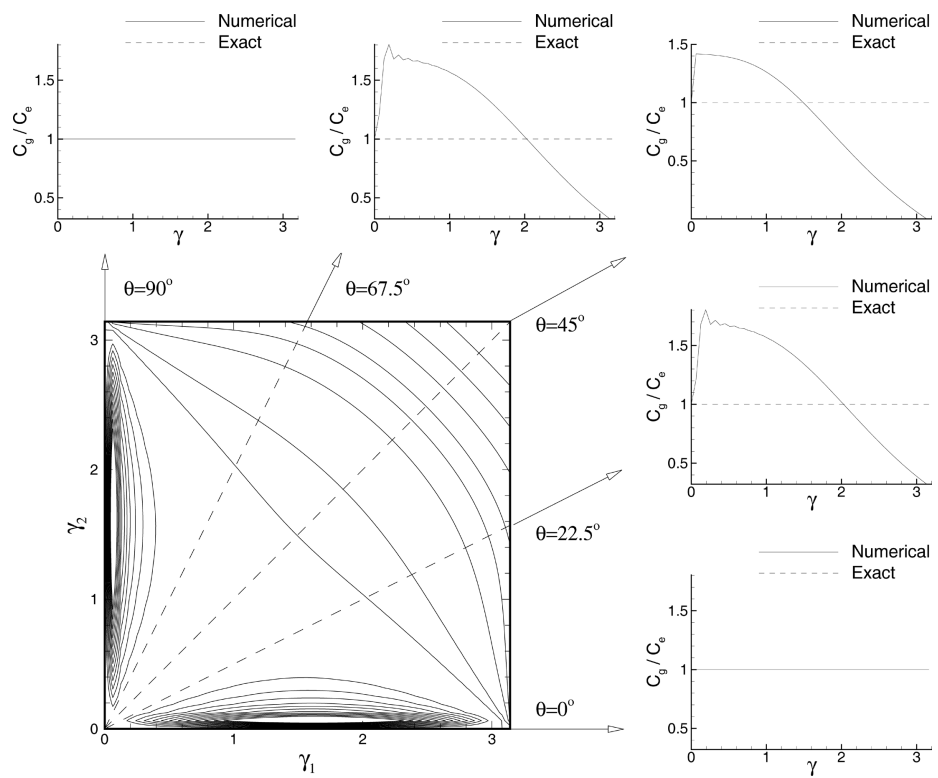

Figure 3. Plots of the group velocity ratio $C_{g} / C_{e}$ against the modified wave number $\left(\gamma_{1}, \gamma_{2}\right)$ at $\mathrm{Pe}_{x}=\mathrm{Pe}_{y}=10^{3}, R_{x}=R_{y}=0$, and $\nu_{x}=v_{y}=0.2$.

$$
\begin{aligned}
\widetilde{\alpha}=\frac{-\mathbf{i}}{h} & \left(a_{1} e^{-\mathbf{i}(\alpha h+\beta h)}+a_{2} e^{-\mathbf{i} \beta h}+a_{3} e^{\mathbf{i}(\alpha h-\beta h)}+a_{4} e^{-\mathbf{i} \alpha h}+a_{5}+a_{6} e^{\mathbf{i} \alpha h}\right. \\
& +a_{7} e^{-\mathbf{i}(\alpha h-\beta h)}+a_{8} e^{\mathbf{i} \beta h}+a_{9} e^{\mathbf{i}(\alpha h+\beta h)} \\
& \left.+a_{10} e^{\mathbf{i}(-\beta h)}+a_{11} e^{\mathbf{i}(\beta h)}+a_{12} e^{\mathbf{i}(-\alpha h)}\right)
\end{aligned}
$$

where $\mathbf{i}=\sqrt{-1}$. Similarly, we can derive

$$
\begin{aligned}
\widetilde{\beta}=\frac{-\mathbf{i}}{h}( & b_{1} e^{-\mathbf{i}(\alpha h+\beta h)}+b_{2} e^{-\mathbf{i} \beta h}+b_{3} e^{\mathbf{i}(\alpha h-\beta h)}+b_{4} e^{-\mathbf{i} \alpha h} \\
& +b_{5}+b_{6} e^{\mathbf{i} \alpha h}+b_{7} e^{-\mathbf{i}(\alpha h-\beta h)}+b_{8} e^{\mathbf{i} \beta h}+b_{9} e^{\mathbf{i}(\alpha h+\beta h)} \\
& \left.+b_{10} e^{\mathbf{i}(-2 \beta h)}+b_{11} e^{\mathbf{i}(2 \beta h)}+b_{12} e^{\mathbf{i}(-2 \alpha h)}\right)
\end{aligned}
$$

To make $\alpha$ be close to $\widetilde{\alpha}$, it is rational to require that $|\alpha h-\widetilde{\alpha} h|^{2}$ ( or the following integrated error $E$ ) should approach zero in the following weak sense [8, 9, 17]:

$$
E(\alpha)=\int_{-\pi / 2}^{\pi / 2} \int_{-\pi / 2}^{\pi / 2}|\alpha h-\widetilde{\alpha} h|^{2} d(\alpha h) d(\beta h)=\int_{-\pi / 2}^{\pi / 2} \int_{-\pi / 2}^{\pi / 2}\left|\mathbf{i} \gamma_{1}-\widetilde{\gamma}_{1}\right|^{2} d \gamma_{1} d \gamma_{2}
$$

where $\left(\gamma_{1}, \gamma_{2}\right)=(\alpha h, \beta h)$. In the discrete system for $\phi_{x}$, the modified wave-number range should be sufficiently wide to define a period of sine (or cosine) wave. This explains why the integral range shown in (28) is chosen to be $-\pi / 2 \leq \gamma_{1}, \gamma_{2} \leq \pi / 2$. 


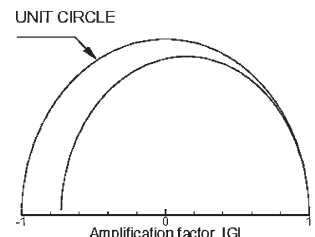

Amplification factor $|\mathrm{G}|$

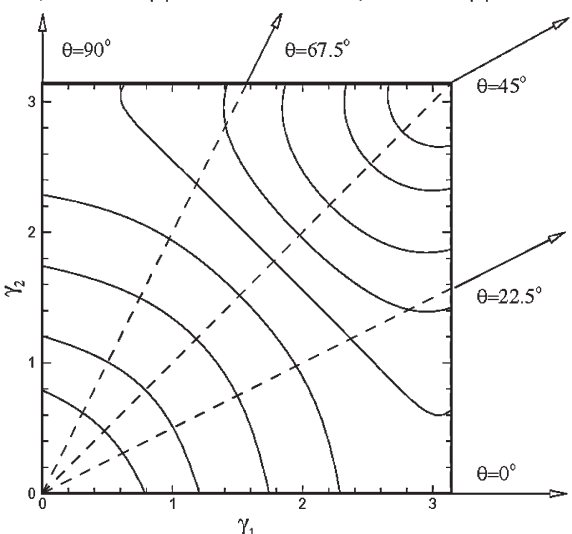

(a)
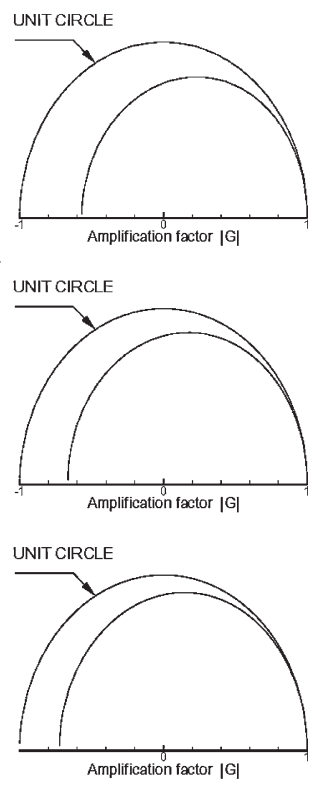

Amplification factor $|G|$

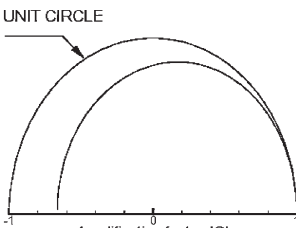

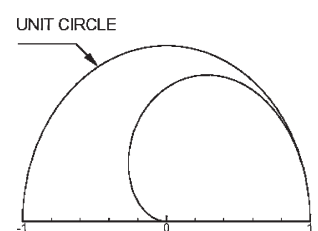

Phase error ratio

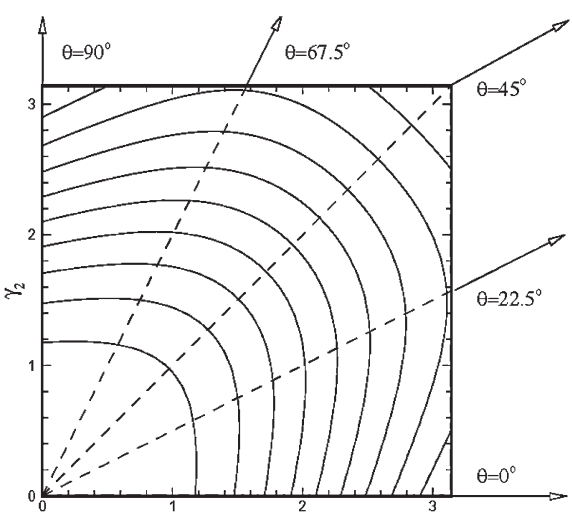

$\gamma_{1}$
UNIT CIRCLE

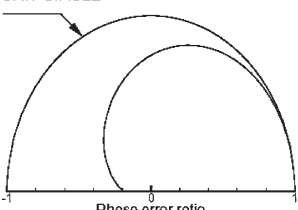

UNIT CIRCLE

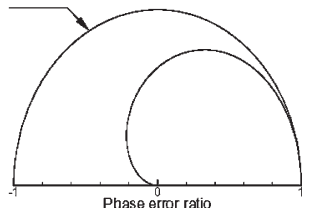

UNIT CIRCLE
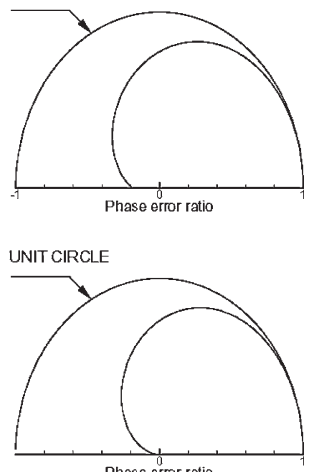

(b)

Figure 4. Plots of $|\mathrm{G}|$ and $\theta / \theta_{e}$ against $\left(\gamma_{1}, \gamma_{2}\right)$ at $\mathrm{Pe}_{x}=\mathrm{Pe}_{y}=10^{3}, R_{x}=R_{y}=0$, and $\nu_{x}=\nu_{y}=0.2:(a)$ amplification factor $|G| ;(b)$ phase angle ratio $\theta / \theta_{e}$. 


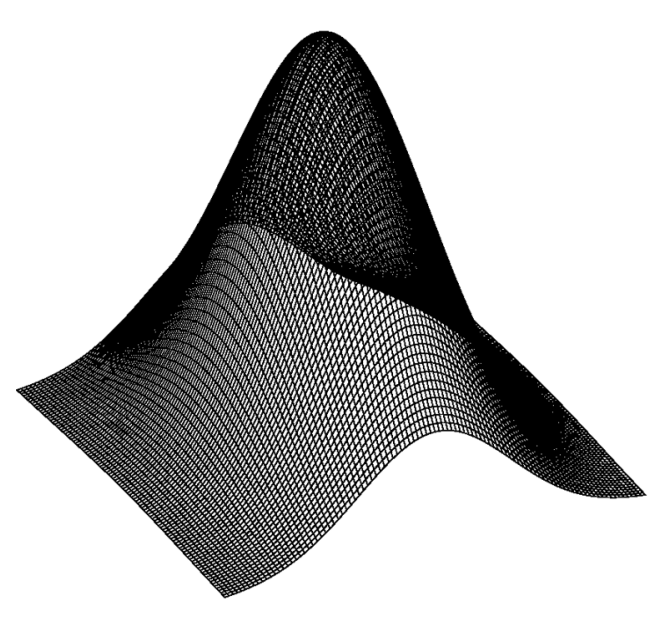

(a)

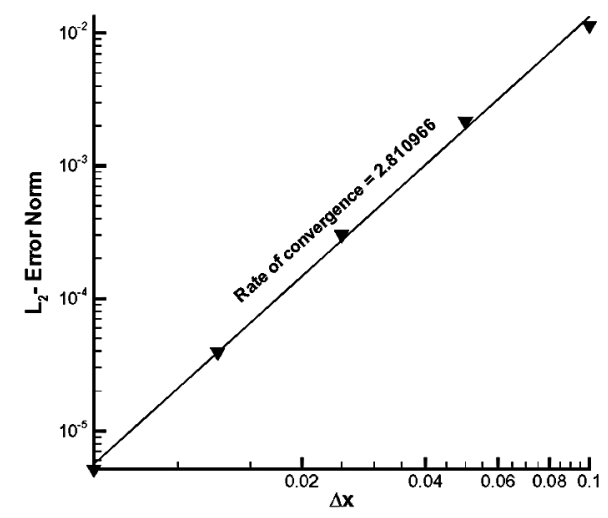

(c)

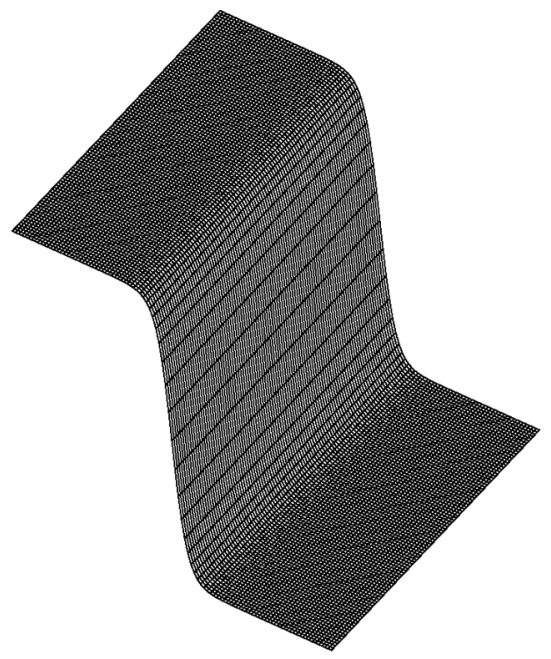

(b)

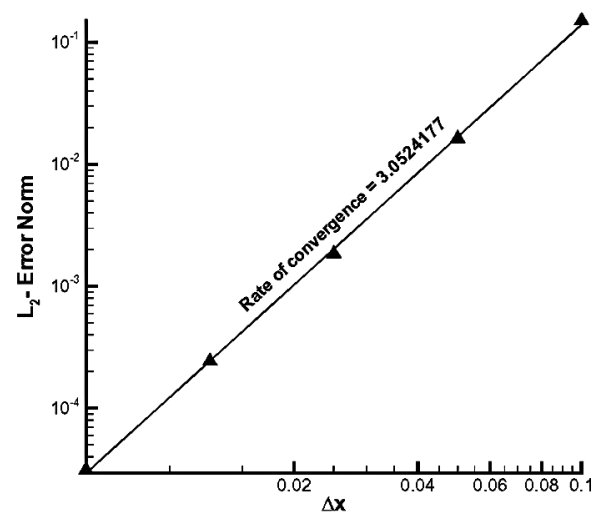

(d)

Figure 5. Simulated solutions for the two problems considered in Section 7.1: (a) simulated Gaussian solution profile; $(b)$ simulated hyperbolic tangent solution profile; $(c)$ rate of convergence for problem $(a) ;(d)$ rate of convergence for problem $(b)$.

To make $E$ a minimum value, we enforce

$$
\frac{\partial E}{\partial a_{6}}=0
$$

With the above extreme condition applied at the point 6 schematic in Figure 1, we are able to obtain the nine introduced coefficients from Eqs. (29) and (12)-(22): 


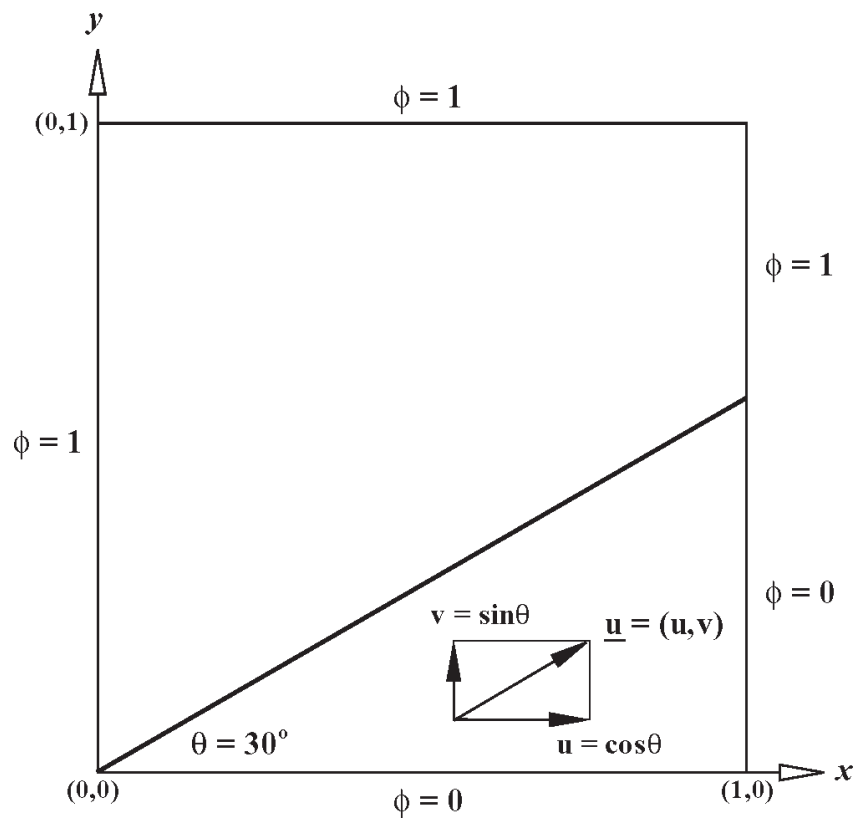

Figure 6. Schematic of the convection-diffusion problem considered in Section 7.2.

$$
\begin{aligned}
& a_{1}=a_{3}=a_{7}=a_{9}=0 \\
& a_{2}=a_{8}=\frac{1}{9} \frac{\pi(3 \pi-10)}{(3 \pi-8)} \\
& a_{4}=-1 \\
& a_{6}=\frac{1}{3} \\
& a_{5}=\frac{1}{6} \frac{3 \pi^{2}-19 \pi+24}{(3 \pi-8)} \\
& a_{10}=a_{11}=-\frac{1}{36} \frac{\pi(3 \pi-10)}{(3 \pi-8)} \\
& a_{12}=\frac{1}{6}
\end{aligned}
$$

The resulting modified equation for $\phi_{x}$ is shown to have spatial accuracy order of three:

$$
\phi_{x} \simeq \frac{h^{3}}{12} \phi_{x x x x}+\frac{h^{3}}{36} \frac{\pi(3 \pi-10)}{(3 \pi+8)} \phi_{y y y y}-\frac{h^{4}}{30} \phi_{x x x x x}+\frac{h^{5}}{72} \phi_{x x x x x x}+\cdots+\text { HOT }
$$

where HOT stands for higher-order terms.

Similarly, for the case of $b>0$, the 13-point stencil approximation equation for $\phi_{y}$ that accommodates the dispersion feature can be derived under the conditions 


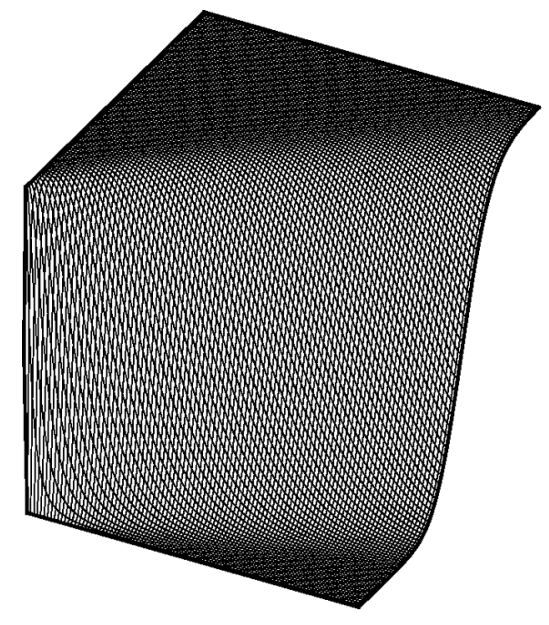

(a)

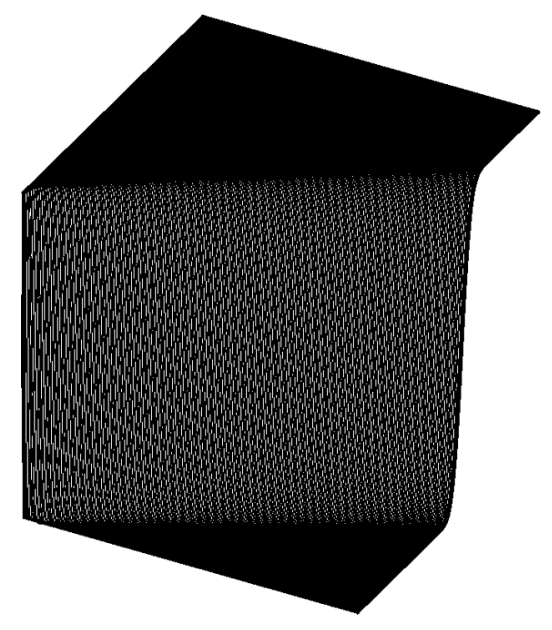

(c)

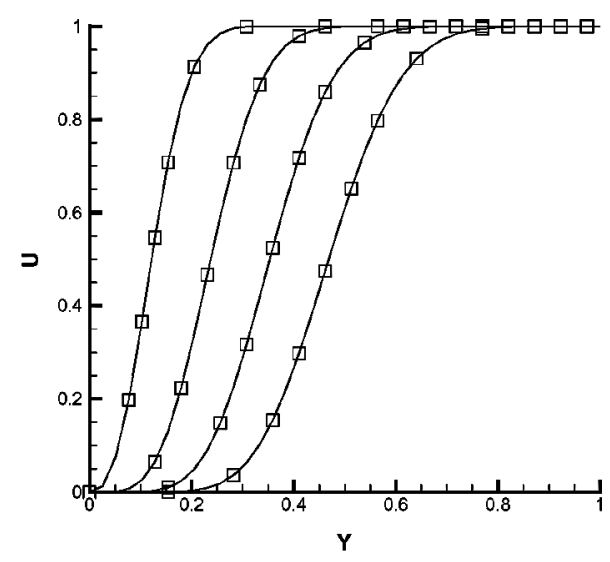

(b)

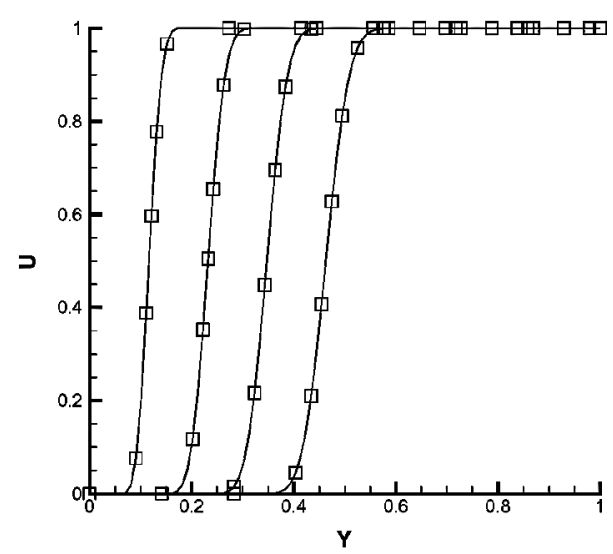

(d) Figure 7. Simulated solution for $\phi$ and their distributions at $x=\frac{1}{5}, \frac{2}{5}, \frac{3}{5}, \frac{4}{5}:(a)-(b) k=10^{-2} ;(c)-(d)$
$k=10^{-3}$.

that $b_{4}=b_{6}=a_{2}, \quad b_{2}=a_{4}, b_{8}=a_{6}, b_{10}=a_{12}, b_{12}=b_{13}=a_{10}, \quad$ and $b_{j}=a_{j}(j=$ $1,3,5,7,9)$. The modified equation for $\phi_{y}$ is derived as

$$
\phi_{y} \simeq \frac{h^{3}}{12} \phi_{y y y y}+\frac{h^{3}}{36} \frac{\pi(3 \pi-10)}{(3 \pi+8)} \phi_{x x x x}-\frac{h^{4}}{30} \phi_{y y y y y}+\frac{h^{5}}{72} \phi_{y y y y y y}+\cdots+\text { HOT }
$$




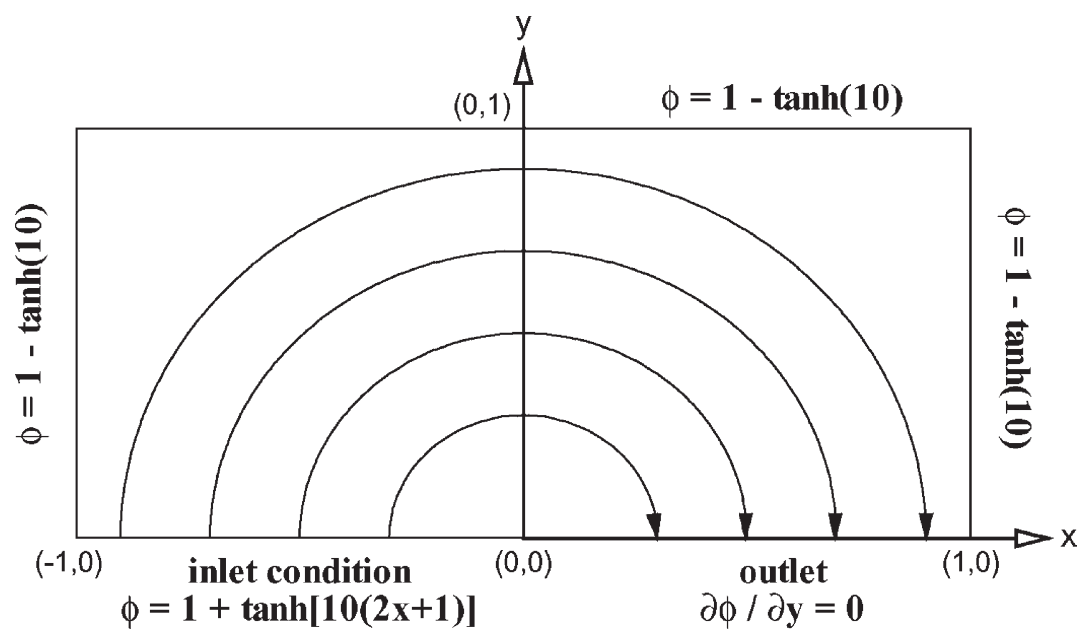

Figure 8. Schematic of the Smith-Hutton problem considered in Section 7.3.

Another choice that can be employed to determine the weighted coefficients $a_{1}-a_{12}$ is to eliminate the term $\phi_{y y y y}$ instead of enforcing $\phi_{x x y y}=0$ for $\phi_{x}$. In the case of $a>0$, we can derive the following coefficients:

$$
\begin{aligned}
& a_{1}=a_{3}=a_{7}=a_{9}=\frac{1}{18} \frac{\pi(3 \pi-10)}{\left(\pi^{2}-6 \pi+8\right)} \\
& a_{2}=a_{8}=-\frac{1}{9} \frac{\pi(3 \pi-10)}{\left(\pi^{2}-6 \pi+8\right)} \\
& a_{4}=-\frac{4}{9} \frac{\left(3 \pi^{2}-16 \pi+18\right)}{\left(\pi^{2}-6 \pi+8\right)} \\
& a_{6}=-\frac{8}{9} \frac{(\pi-3)}{\left(\pi^{2}-6 \pi+8\right)} \\
& a_{5}=\frac{1}{18} \frac{21 \pi^{2}-94 \pi+72}{\left(\pi^{2}-6 \pi+8\right)} \\
& a_{10}=a_{11}=0 \\
& a_{12}=\frac{1}{6}
\end{aligned}
$$

The corresponding modified equation for $\phi_{x}$ can be also shown to have spatial accuracy order of three:

$$
\phi_{x} \simeq \frac{h^{3}}{12} \phi_{x x x x}+\frac{h^{3}}{18} \frac{\pi(3 \pi-10)}{\left(\pi^{2}-6 \pi+8\right)} \phi_{x x y y}-\frac{h^{4}}{30} \phi_{x x x x x}+\frac{h^{5}}{72} \phi_{x x x x x x}+\cdots+\text { HOT }
$$




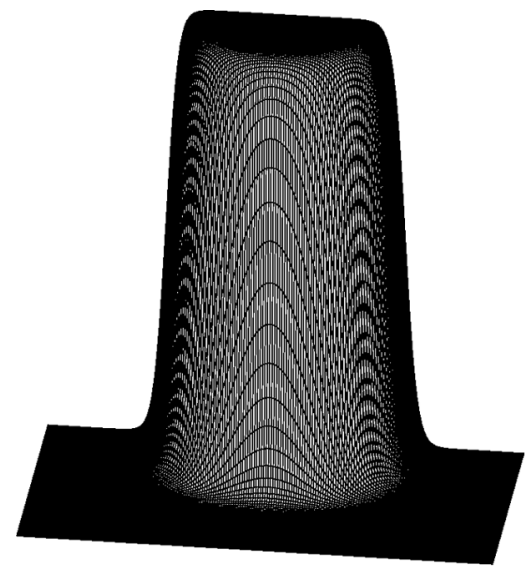

(a)

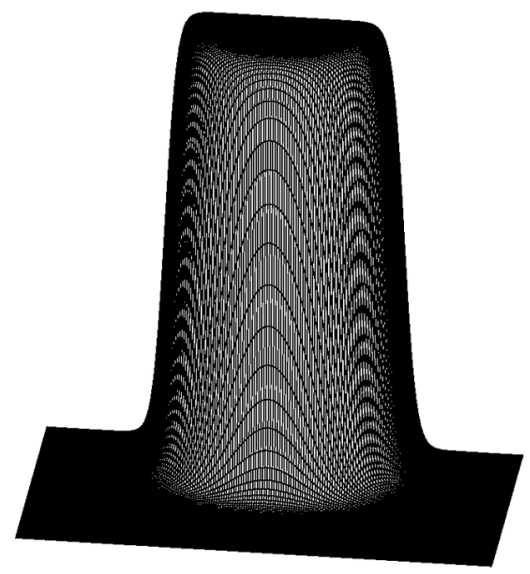

(c)

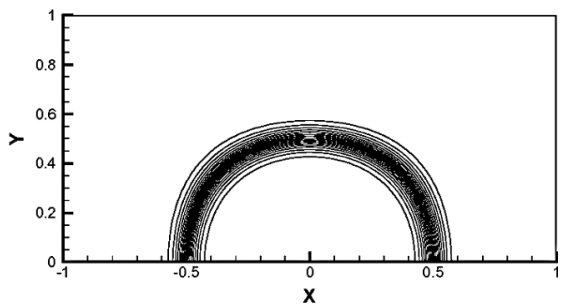

(b)

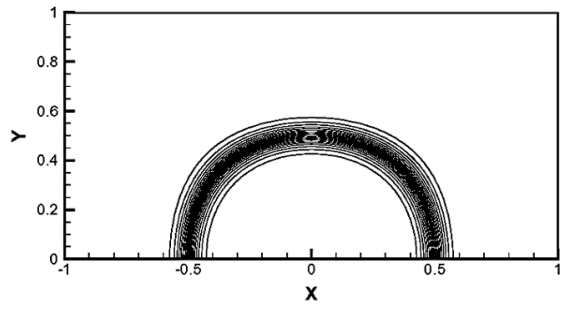

(d)

Figure 9. Simulated $\phi$ at different diffusivities $k$ for the problem considered in Section 7.3 using the DRP scheme with coefficients given in Eqs. (30)-(36): (a)-(b) $k=10^{-12} ;(c)-(d) k=10^{-14}$.

It is found that the coefficient of the scheme's leading error term is larger than that with the weighted coefficients given in (30)-(36). In what follows, we will consider the DRP scheme for $\phi_{x}$ by Eqs. (10) and (30)-(36).

\section{DISPERSION AND VON NEUMANN STABILITY ANALYSES}

\subsection{Dispersive Analysis}

Fundamental study of the two-dimensional linear scalar CDR equation will be conducted within the implicit Euler time-stepping framework: 


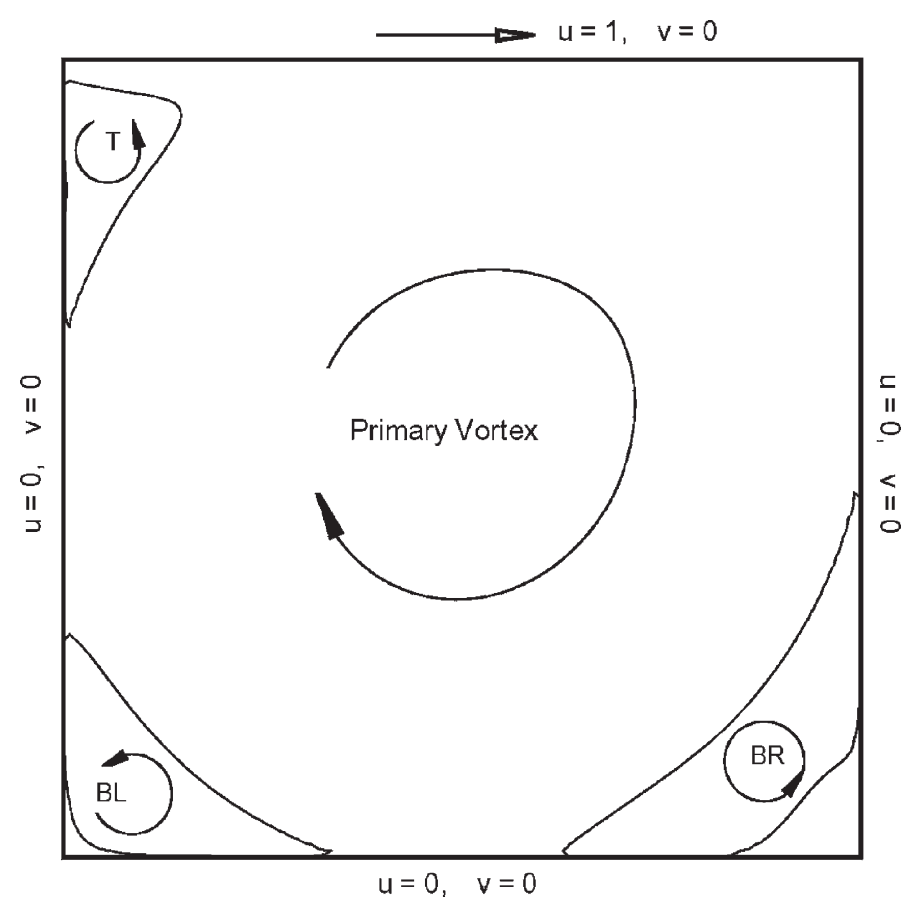

Figure 10. Schematic of the eddy centers in the lid-driven cavity.

$$
\frac{\phi^{n+1}-\phi^{n}}{\Delta t}+a \phi_{x}+b \phi_{y}-k \nabla^{2} \phi+c \phi=0
$$

Given $\phi(x, y, t=0)=\exp [\mathbf{i}(\alpha x+\beta y)]$, the exact solution for Eq. (46) can be easily derived in terms of $(\alpha, \beta)$ :

$$
\phi(x, y, t)=\exp \left\{-\left[k\left(\alpha^{2}+\beta^{2}\right)+c\right] t\right\} \exp \{\mathbf{i}[\alpha(x-a t)+\beta(y-b t)]\}
$$

The 13-point stencil discrete equation at an interior point $(i, j)$ is assumed to take the following form:

$$
\begin{aligned}
& A_{1} \phi_{i-1, j-1}^{n+1}+A_{2} \phi_{i, j-1}^{n+1}+A_{3} \phi_{i+1, j-1}^{n+1}+A_{4} \phi_{i-1, j}^{n+1}+A_{5} \phi_{i, j}^{n+1} \\
& \quad+A_{6} \phi_{i+1, j}^{n+1}+A_{7} \phi_{i-1, j+1}^{n+1}+A_{8} \phi_{i, j+1}^{n+1}+A_{9} \phi_{i+1, j+1}^{n+1} \\
& \quad+A_{10} \phi_{i, j-2}^{n+1}+A_{11} \phi_{i, j+2}^{n+1}+A_{12} \phi_{i-2, j}^{n+1}+A_{13} \phi_{i+2, j}^{n+1}=\phi_{i, j}^{n}
\end{aligned}
$$

The exact solution for Eq. (46) is given below to account for the inevitable amplitude and phase errors: 


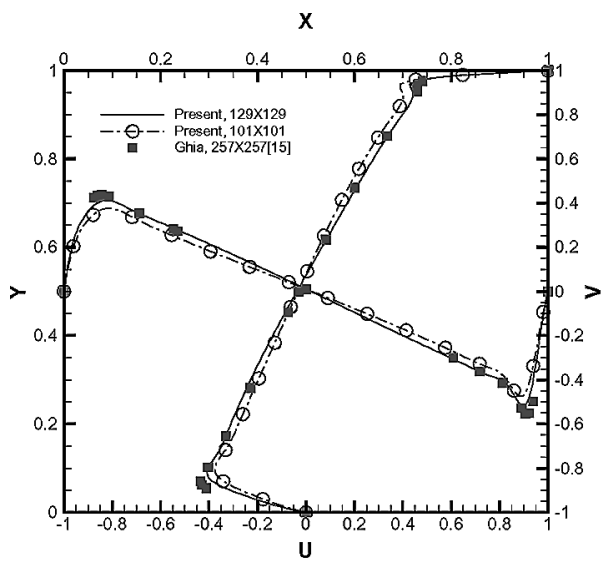

(a)

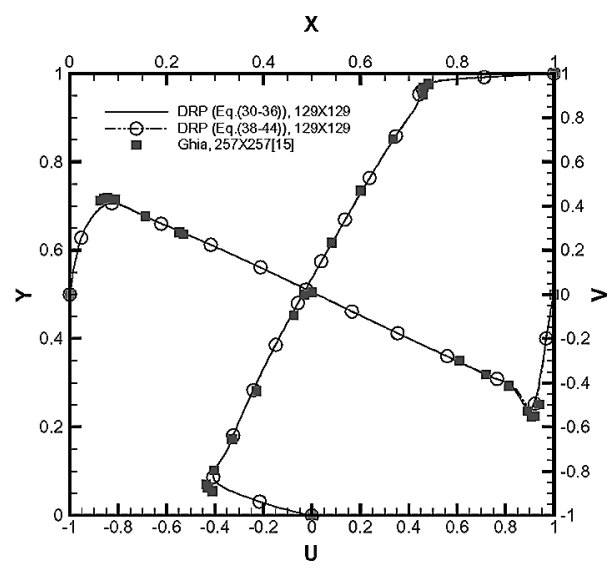

(b)

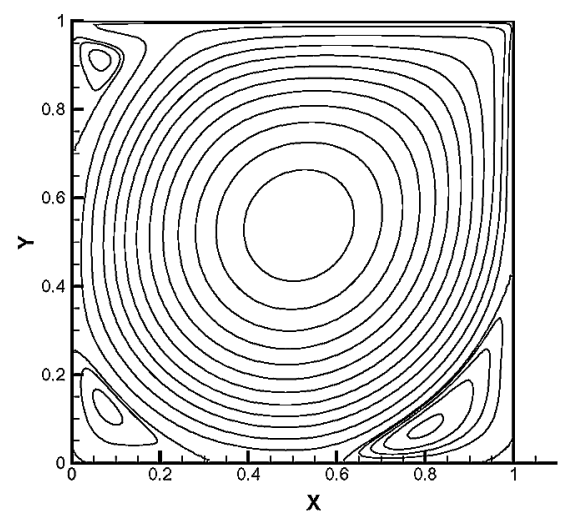

(c)

Figure 11. Comparison of the simulated and Ghia's velocity profiles $u(x, 0.5)$ and $v(0.5, y)$ at $\operatorname{Re}=5,000$ : (a) solutions obtained at different mesh sizes; $(b)$ solutions obtained by DRP scheme for coefficients given in Eqs. (30)-(36) and DRP scheme for coefficients given in Eqs. (38)-(44); (c) simulated contours of stream function for the lid-driven cavity problem investigated at $\mathrm{Re}=5,000$.

$$
\begin{aligned}
\widetilde{\phi}(x, y, t)= & \exp \left\{-\left[\left(k \alpha^{2}+\frac{c}{2}\right) \frac{k_{r}}{\gamma_{1}^{2}}+\left(k \beta^{2}+\frac{c}{2}\right) \frac{k_{r}}{\gamma_{2}^{2}}\right] t\right\} \\
& \times \exp \left\{\mathbf{i}\left[\alpha\left(x-a \frac{k_{i}}{\gamma_{1}} t\right)+\beta\left(y-b \frac{k_{i}}{\gamma_{2}} t\right)\right]\right\}
\end{aligned}
$$

or

$$
\widetilde{\phi}(x, y, t)=A \exp (\bar{p}+\mathbf{i} \bar{q}) \exp \left[\mathbf{i} k_{m}(x+y)\right]
$$


Table 1. Comparison of the simulated eddy centers (primary eddy $\mathrm{P}$, corner eddies BL and BR, and eddy $\mathrm{T}$ near the cavity roof) with those reported in [23] for the cases with $\mathrm{Re}=400,1,000,3,200$, and 5,000

\begin{tabular}{llcccc}
\hline & & \multicolumn{5}{c}{$\mathrm{Re}$} \\
\cline { 3 - 6 } Symbol & Authors & 400 & 1,000 & 3,200 & 5,000 \\
\hline Primary & Present & $0.5599,0.6062$ & $0.5332,0.5658$ & $0.5180,0.5453$ & $0.5138,0.5356$ \\
& Ghia & $0.5547,0.6055$ & $0.5313,0.5625$ & $0.5165,0.5469$ & $0.5117,0.5352$ \\
$\mathrm{~T}$ & Present & - & - & $0.0532,0.8965$ & $0.0643,0.9149$ \\
& Ghia & - & - & $0.0547,0.8984$ & $0.0625,0.9141$ \\
BL & Present & $0.0495,0.0468$ & $0.0846,0.0787$ & $0.0838,0.1102$ & $0.0730,0.1337$ \\
& Ghia & $0.0508,0.0469$ & $0.0859,0.0781$ & $0.0859,0.1094$ & $0.0703,0.1367$ \\
BR & Present & $0.8859,0.1235$ & $0.8640,0.1098$ & $0.8203,0.0876$ & $0.8000,0.0760$ \\
& Ghia & $0.8906,0.1250$ & $0.8594,0.1094$ & $0.8125,0.0859$ & $0.8086,0.0742$ \\
Mesh points & Present & 101 & 129 & 129 & 129 \\
& Ghia & 257 & 129 & 129 & 257 \\
\hline
\end{tabular}

With $\left(\gamma_{1}, \gamma_{2}\right)=(\alpha h, \beta h)$ defined in Section 5, where $\nu_{x}=a \Delta t / h, v_{y}=b \Delta t / h$, $\mathrm{Pe}_{x}=a h / k, \mathrm{Pe}_{y}=b h / k, R_{x}=c h / a$, and $R_{y}=c h / b$, the following equation can be easily desired:

$$
\begin{gathered}
-\left\{\left[\left(k \alpha^{2}+\frac{c}{2}\right) \frac{k_{r}}{\gamma_{1}^{2}}+\left(k \beta^{2}+\frac{c}{2}\right) \frac{k_{r}}{\gamma_{2}^{2}}\right]+\mathbf{i}\left(\alpha a \frac{k_{i}}{\gamma_{1}}+\beta b \frac{k_{i}}{\gamma_{2}}\right)\right\} \Delta t \\
=-\left\{\left[\left(k \alpha^{2}+\frac{c}{2}\right) \frac{k_{r}}{(\alpha h)^{2}}+\left(k \beta^{2}+\frac{c}{2}\right) \frac{k_{r}}{(\beta h)^{2}}\right]\right. \\
\left.+\mathbf{i}\left(\alpha a \frac{k_{i}}{\alpha h}+\beta b \frac{k_{i}}{\beta h}\right)\right\} \Delta t
\end{gathered}
$$

Dispersion analysis of the discrete equation for (46) involves substituting $\phi_{i, j}$, $\phi_{i \pm 1, j}, \phi_{i, j \pm 1}, \phi_{i \pm 2, j}, \phi_{i, j \pm 2}$, and $\phi_{i \pm 1, j \pm 1}$, which are obtained from Eq. (49), into Eq. (46). After some algebra, $k_{r}$ and $k_{i}$ for the respective amplitude and phase errors are derived as

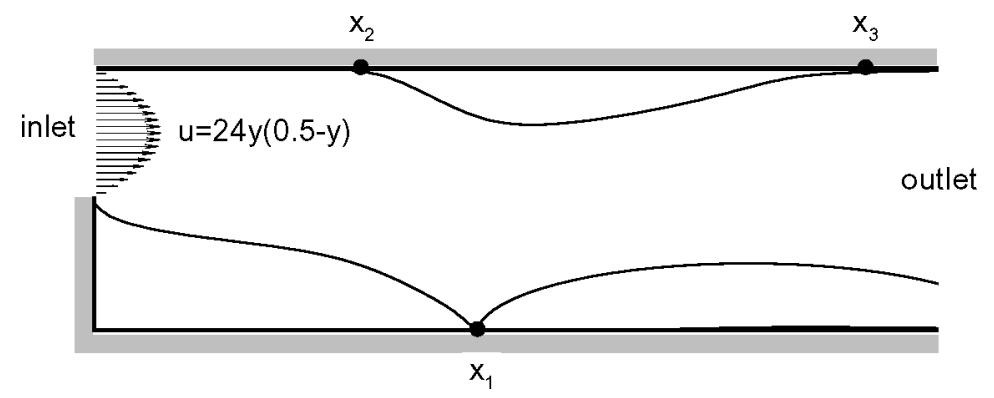

Figure 12. Schematic of the backward-facing-step flow problem. 
Table 2. Simulated reattachment and separation points $x_{1}, x_{2}, x_{3}$, schematic in Figure 12, for the benchmark backward-facing-step flow problem (Note that DRP and DRP* correspond to the schemes with weighting coefficients Eqs. (30)-(36) and Eqs. (38)-(44), respectively)

\begin{tabular}{lllr}
\hline & $x_{1} / h$ & $x_{2} / h$ & $x_{3} / h$ \\
\hline Gartling [25] & 6.10 & 4.85 & 10.48 \\
Betts and Sayma [24] & 5.605 & 4.40 & 10.43 \\
Srinivasan and Rubin [24] & 6.22 & 5.09 & 10.25 \\
Barton [24] & 6.015 & 4.82 & 10.48 \\
Present work, DRP (Eq. (30-36)) & 5.97 & 4.88 & 10.21 \\
Present work, DRP* (Eq. (38-44)) & 5.93 & 4.83 & 10.19 \\
\hline
\end{tabular}

$$
\begin{gathered}
k_{r}=\frac{-\bar{p}}{\left(\nu_{x} / \mathrm{Pe}_{x}\right)+\left(\nu_{x} / \mathrm{Pe}_{x}\right)+\frac{1}{2}\left\{\left[\frac{\left(\nu_{x} R_{x}+1\right)}{\gamma_{1}^{2}}\right]+\left[\frac{\left(\nu_{y} R_{y}+1\right)}{\gamma_{2}^{2}}\right]\right\}} \\
k_{i}=\frac{-\bar{q}}{\nu_{x}+\nu_{y}}
\end{gathered}
$$

The derivation is followed by substituting the exact expressions for $\phi_{i, j}, \phi_{i \pm 1, j}, \phi_{i, j \pm 1}$, $\phi_{i \pm 2, j}, \phi_{i, j \pm 2}, \phi_{i \pm 1, j \pm 1}$ into Eq. (48) to yield:

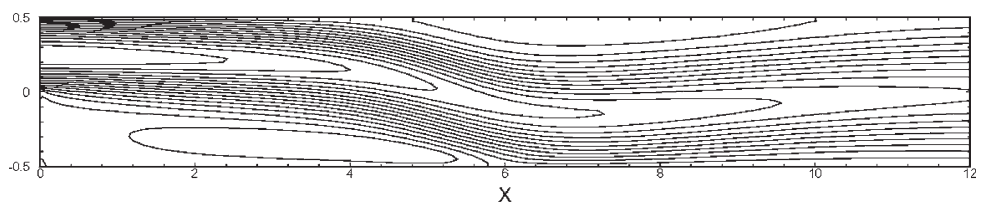

(a)

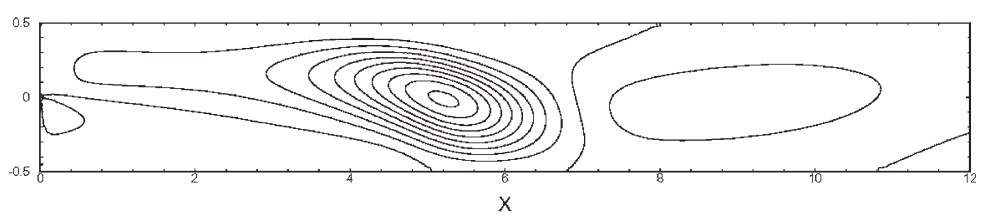

(b)

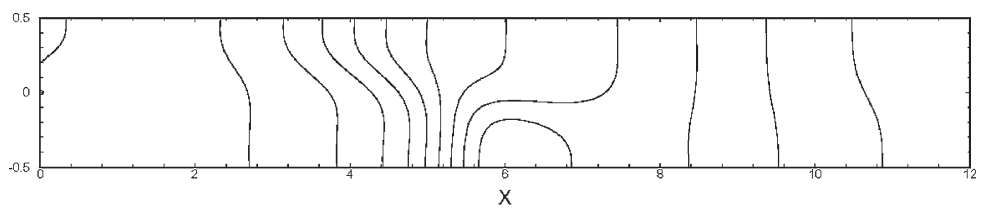

(c)

Figure 13. Simulation results for the backward-facing-step flow problem investigated at $\operatorname{Re}=800$ : (a) $u$ contours; (b) $v$ contours; (c) $p$ contours. 


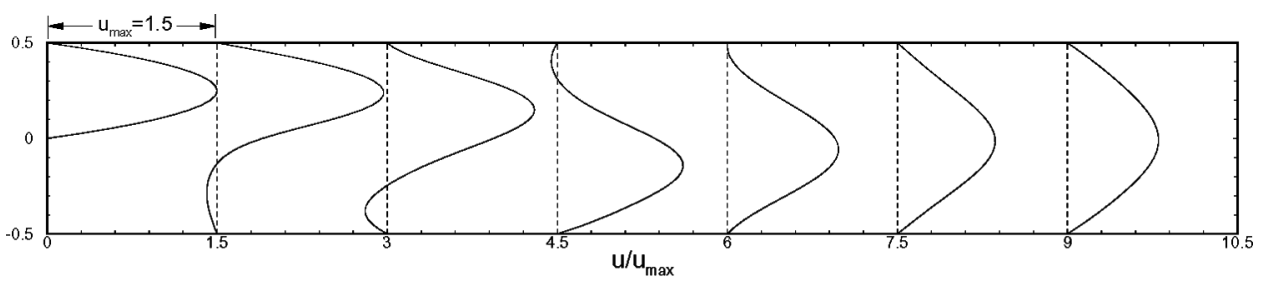

(a)

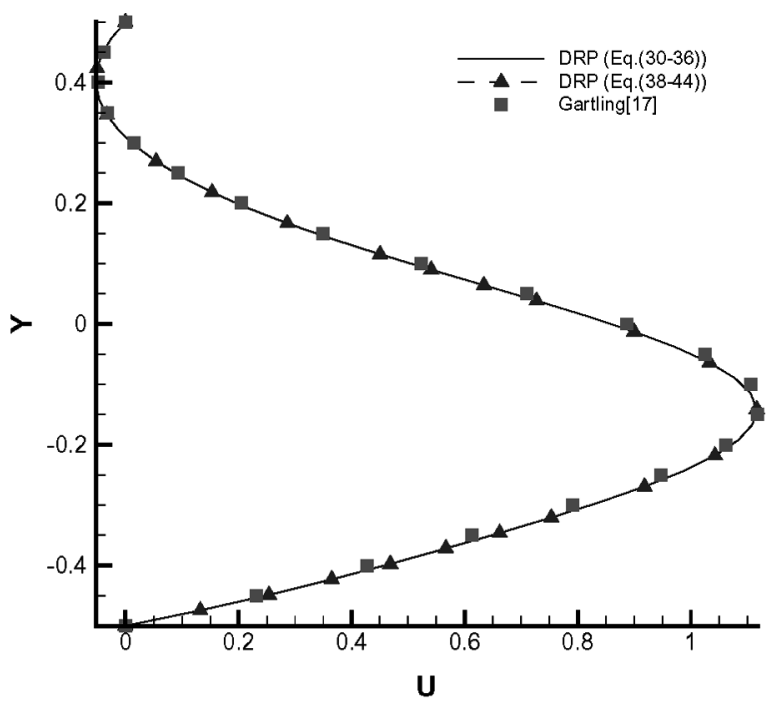

(b)

Figure 14. Simulated velocity profiles for the backward-facing-step flow problem investigated at $\mathrm{Re}=$ 800: $(a) u$ profile; (b) comparison between the two DRP schemes for coefficients given in Eqs. (30)-(36), Eqs. (38)-(44), and Gartling solutions.

$$
\begin{aligned}
& e^{\bar{p}}\left(A_{1} e^{\mathbf{i}(\bar{q}-x)}+A_{2} e^{\mathbf{i}\left(\bar{q}-\gamma_{2}\right)}+A_{3} e^{\mathbf{i}(\bar{q}+y)}+A_{4} e^{\mathbf{i}\left(\bar{q}-\gamma_{1}\right)}+A_{5} e^{\mathbf{i} q}\right. \\
& \quad+A_{6} e^{\mathbf{i}\left(\bar{q}+\gamma_{1}\right)}+A_{7} e^{\mathbf{i}(\bar{q}-y)}+A_{8} e^{\mathbf{i}\left(\bar{q}+\gamma_{2}\right)}+A_{9} e^{\mathbf{i}(\bar{q}+x)}+A_{10} e^{\mathbf{i}\left(\bar{q}-2 \gamma_{2}\right)} \\
& \left.\quad+A_{11} e^{\mathbf{i}\left(\bar{q}+2 \gamma_{2}\right)}+A_{12} e^{\mathbf{i}\left(\bar{q}-2 \gamma_{1}\right)}+A_{13} e^{\mathbf{i}\left(\bar{q}+2 \gamma_{1}\right)}\right)=1
\end{aligned}
$$

where $x=\gamma_{1}+\gamma_{2}$ and $y=\gamma_{1}-\gamma_{2}$. In the above, $q$ and $p$ can be derived respectively from the imaginary and real parts as follows:

$$
\bar{q}=\tan ^{-1} \frac{n}{m}
$$




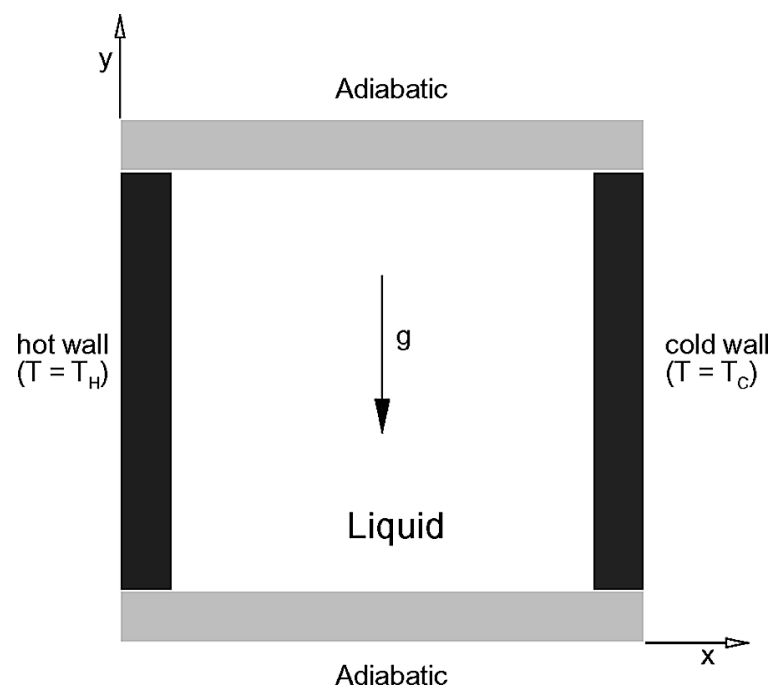

Figure 15. Schematic of the natural-convection problem.

$$
p=\ln \left\{\frac{1}{m \cos q+n \sin q}\right\}
$$

where

$$
\begin{aligned}
m= & \left(A_{1}+A_{9}\right) \cos x+\left(A_{2}+A_{8}\right) \cos \gamma_{2}+\left(A_{3}+A_{7}\right) \cos y+\left(A_{4}+A_{6}\right) \cos \gamma_{1}+A_{5} \\
& +\left(A_{10}+A_{11}\right) \cos \left(2 \gamma_{2}\right)+\left(A_{12}+A_{13}\right) \cos \left(2 \gamma_{1}\right) \\
& \\
& \quad\left(A_{1}-A_{9}\right) \sin x+\left(A_{2}-A_{8}\right) \sin \gamma_{2}+\left(A_{3}-A_{7}\right) \sin y+\left(A_{4}-A_{6}\right) \sin \gamma_{1} \\
& +\left(A_{10}-A_{11}\right) \sin \left(2 \gamma_{2}\right)+\left(A_{12}-A_{13}\right) \sin \left(2 \gamma_{1}\right)
\end{aligned}
$$

It is observed from Figure 2, which plots $k_{r}$ and $k_{i}$ against $\left(v_{x}, v_{y}\right)$ and $\left(\mathrm{Pe}_{x}, \mathrm{Pe}_{y}\right)$ [fixed $\left.\left(R_{x}, R_{y}\right)\right]$, that $k_{i}$ agrees perfectly with $\left(\gamma_{1}, \gamma_{2}\right)$ in the small wave-number range. The larger the wave numbers, the less satisfactory is the predicted phase. In contrast to $k_{i}$, the amplitude error is exhibited even in the small wave-number range. In Figure 3, we plot the ratio of numerical group velocity $C_{g}\left\{\equiv 1 / 2\left[\left(d \omega / d \gamma_{1}\right)+\left(d \omega / d \gamma_{2}\right)\right]\right\}$ with respect to the analytical wave velocity, where $\omega\left[\equiv a \alpha\left(k_{i} / \gamma_{1}\right)+b \beta\left(k_{i} / \gamma_{2}\right)\right]$ is obtained from the dispersion equation.

\subsection{Fourier or von Neumann Stability Analysis}

We also conduct Fourier (or von Neumann) stability analysis [19]. Let $\chi_{x}=\chi_{y}=(2 \pi m / 2 L) h(m=0,1,2,3, \ldots, M), h$ be the grid size, and $2 L$ be the period of fundamental frequency $(m=1)$. Then the amplification factor 


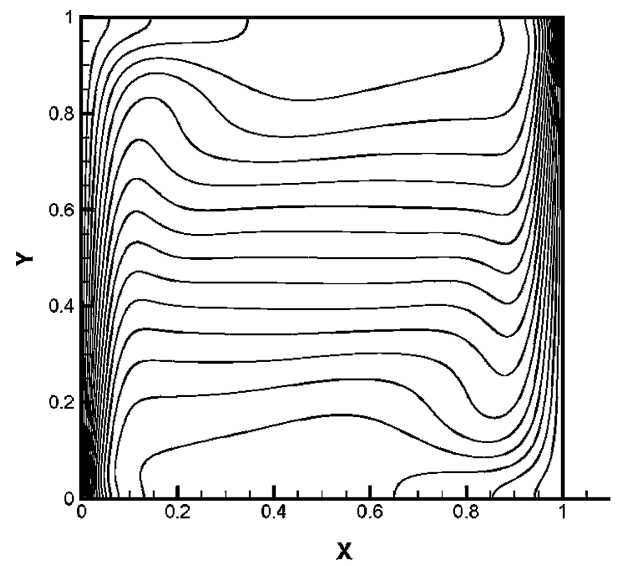

(a)

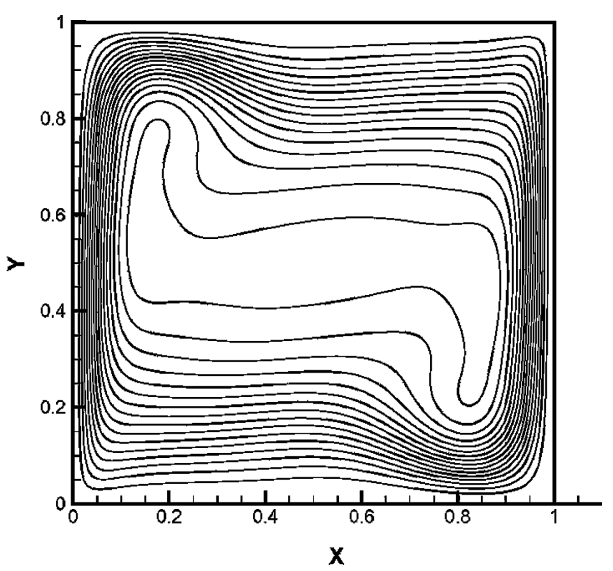

(b)

Figure 16. Simulated results for the natural-convection problem investigated at $\mathrm{Ra}=10^{6}:(a)$ temperature contours; (b) stream function contours.

$G\left(\equiv \phi_{i, j}^{n+1} / \phi_{i, j}^{n}\right)$ can be derived as

$$
G=e^{\bar{p}}(\cos \bar{q}+\mathbf{i} \sin \bar{q})
$$

As seen in Figure $4 a$, the magnitude of $|G|$ is always smaller than one. The proposed scheme is, thus, unconditionally stable. The amplification factor shown in (59) can be rewritten in exponential form as $G=|G| e^{\mathbf{i} \theta}$, where $\theta$ is the phase angle:

$$
\theta=\tan ^{-1}\left|\frac{\operatorname{Im}(G)}{\operatorname{Re}(G)}\right|
$$

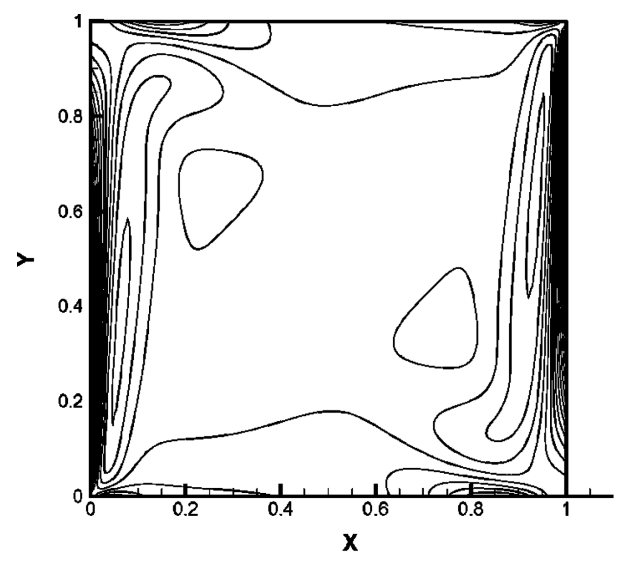

(a)

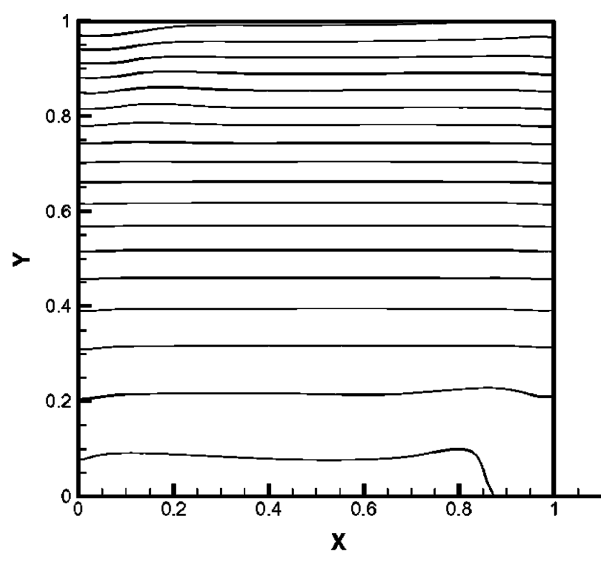

(b)

Figure 17. Simulated results for the natural-convection problem considered at $\mathrm{Ra}=10^{6}$ : $(a)$ vorticity contours; (b) pressure contours. 


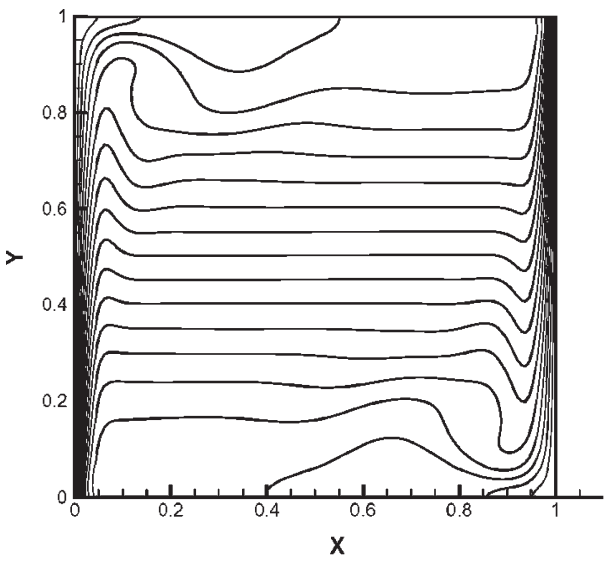

(a)

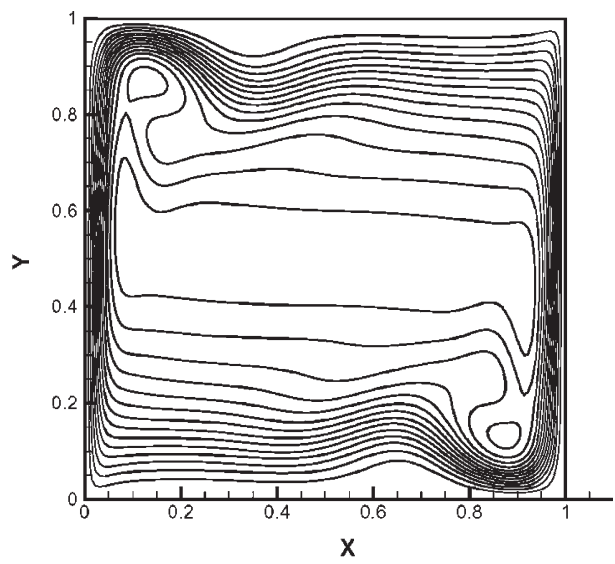

(b)

Figure 18. Simulated results for the natural-convection problem considered at $\mathrm{Ra}=10^{7}:(a)$ temperature contours; (b) stream function contours.

The exact phase angle $\theta_{e}$ can be derived as $-\left(\chi_{x} \nu_{x}+\chi_{y} \nu_{y}\right)$. Therefore, we can derive the following relative phase shift error:

$$
\frac{\theta}{\theta_{e}}=\frac{\tan ^{-1}|\operatorname{Im}(G) / \operatorname{Re}(G)|}{-\left(\chi_{x} \nu_{x}+\chi_{y} \nu_{y}\right)}
$$

For clearness, $\theta / \theta_{e}$ is plotted against $\left(\chi_{x}, \chi_{y}\right),\left(\nu_{x}, \nu_{y}\right),\left(\mathrm{Pe}_{x}, \mathrm{Pe}_{y}\right)$, and $\left(R_{x}, R_{y}\right)$ in Figure $4 b$.

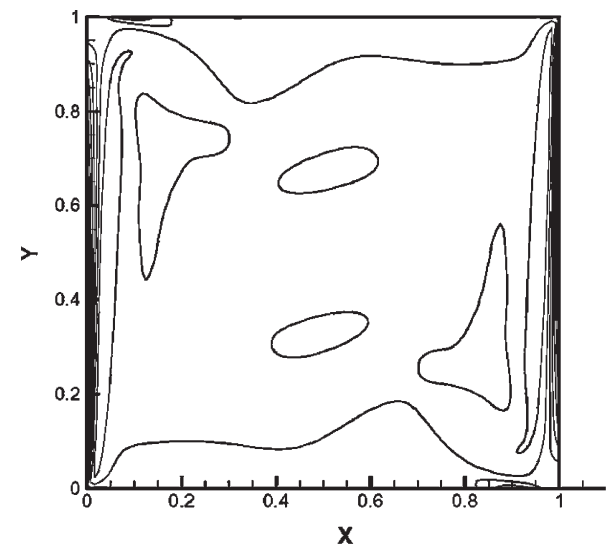

(a)

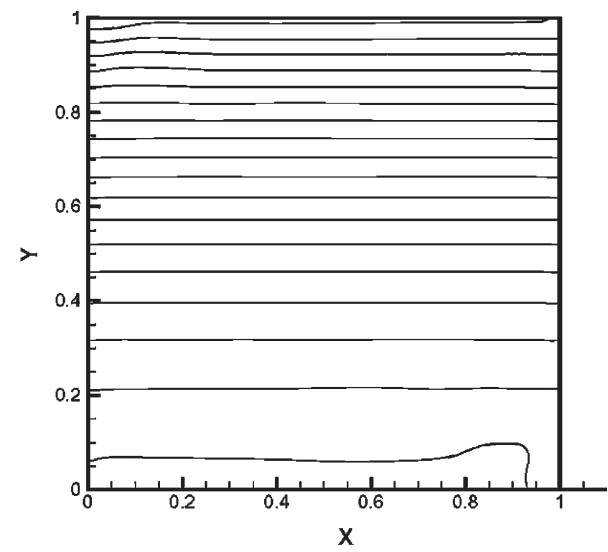

(b)

Figure 19. Simulated results for the natural-convection problem considered at $\mathrm{Ra}=10^{7}$ : $(a)$ vorticity contours; $(b)$ pressure contours. 


\section{NUMERICAL STUDIES}

\subsection{Gaussian and Hyperbolic Tangent Problem}

The model equation (9) in $[0,1] \times[0,1]$ is solved at $(a, b)=(1,0), k=10^{-10}$ and $c=0$. Two source terms $f$ are specified as follows to render the respective exact solutions [20]:

$$
\begin{gathered}
\phi(x, y)=\exp \left(-\frac{(x-0.5)^{2}}{0.2}-\frac{3(y-0.5)^{2}}{0.2}\right) \\
\phi(x, y)=\frac{1}{2}\left[1-\tanh \left(\frac{x-0.5}{0.05}\right)\right]
\end{gathered}
$$

Simulations were carried out at $\Delta x=\Delta y=1 / 10,1 / 20,1 / 40,1 / 80$, and $1 / 160$ to calculate the finite-difference errors cast in $L_{2}$-norm form. This is followed by plotting in Figure 5 the value of $\log \left(\operatorname{err}_{1} / \operatorname{err}_{2}\right)$ against $\log \left(h_{1} / h_{2}\right)$, where $\operatorname{err}_{1}$ and $\operatorname{err}_{2}$ error norms are obtained at the consecutively refined mesh sizes $h_{1}$ and $h_{2}$, to calculate the scheme's rate of convergence. Good agreement with the exact solutions and good rate of convergence are both demonstrated in the simulated solutions.

\subsection{Skew Convection-Diffusion Problem [21]}

In Figure 6, the cavity of unit length is divided into two by the straight line that passes through $(0,0)$ and has a slope of $\tan ^{-1}(b / a)$, where $a$ and $b$ are shown in Eq. (9). We consider here the unit velocity vector $(a, b)$, which is parallel to the dividing line, in $121 \times 121$ and $201 \times 201$ uniformly discretized mesh systems for fluids with $k=10^{-2}$ and $k=10^{-3}$, respectively. Subject to the boundary condition for the working variable, a shear layer is seen in the vicinity of the dividing line. In Figure 7, no oscillatory solution is found to occur in regions near and away from the dividing line for $k$ with its value as small as $10^{-3}$.

\subsection{Convection-Diffusion Problem of Smith and Hutton}

The problem of Smith and Hutton [22] is investigated at $a=2 y\left(1-x^{2}\right)$ and $b=-2 x\left(1-y^{2}\right)$. Along the inlet schematic in Figure $8, \phi$ is prescribed as follows:

$$
\phi(-1 \leq x \leq 0, y=0)=1+\tanh [(2 x+1) \times 10]
$$

Along $x=-1, y=1$ and $x=1$, we prescribe $\phi=1-\tanh (10)$, while at the outlet $(0 \leq x \leq 1, y=0)$, a zero gradient condition is specified for $\phi$. For the cases with $k=10^{-14}$ and $10^{-12}$, the results investigated at $\Delta x=\Delta y=10^{-2}$ are shown in Figure 9 to reveal the efficacy of the proposed DRP method. 
Table 3. Comparison of the simulated streamfunctions at $(0.5,0.5)$ with other solutions reported in [26]

\begin{tabular}{|c|c|c|c|c|c|}
\hline $\mathrm{Ra}$ & $10^{3}$ & $10^{4}$ & $10^{5}$ & $10^{6}$ & $10^{7}$ \\
\hline De Vahl Davis & 1.174 & 5.071 & 9.111 & 16.32 & \\
\hline Ramaswamy, Jue, and Akin & 1.170 & 5.099 & 9.217 & 16.68 & 29.436 \\
\hline Le Quéré & & & & 16.38 & 29.362 \\
\hline Dennis and Hudson & 1.175 & 5.074 & 9.113 & & \\
\hline Kalita, Dalal, and Dass & 1.175 & 5.080 & 9.123 & 16.42 & 29.382 \\
\hline Present work & 1.175 & 5.071 & 9.113 & 16.42 & 29.432 \\
\hline
\end{tabular}

Table 4. Comparison of the simulated $V_{\max }$ at $(0.5, y)$ with other solutions reported in [26]

\begin{tabular}{lccccc}
\hline Ra & $10^{3}$ & $10^{4}$ & $10^{5}$ & $10^{6}$ & $10^{7}$ \\
\hline Chenoweth and Paolucci & 3.695 & 19.62 & 68.63 & 220.8 & 699.0 \\
De Vahl Davis & 3.697 & 19.62 & 68.63 & 219.4 & 717.04 \\
Ramaswamy, Jue, and Akin & & 19.62 & 68.64 & 232.97 & 699.2 \\
Le Quéré & & & & 220.56 & 705.3 \\
Saitoh and Hirosh & 3.697 & 19.62 & & 216.76 & \\
Ho and Lin & & 19.63 & 68.63 & 219.86 & 220.46 \\
Hortmann, Peric, and Scheure & 3.698 & 19.63 & 68.64 & & 696.2 \\
Dennis and Hudson & 3.697 & 19.61 & 68.61 & 221.66 & 695.6 \\
Kalita, Dalal, and Dass & 3.695 & 19.61 & 68.55 & 219.88 & \\
Present work & & & & & \\
\hline
\end{tabular}

Table 5. Comparison of the simulated $\overline{\mathrm{Nu}}$ with other solutions reported in [26]

\begin{tabular}{|c|c|c|c|c|c|}
\hline $\mathrm{Ra}$ & $10^{3}$ & $10^{4}$ & $10^{5}$ & $10^{6}$ & $10^{7}$ \\
\hline Chenoweth and Paolucci & 1.118 & 2.244 & 4.520 & 8.822 & 16.82 \\
\hline De Vahl Davis & 1.118 & 2.243 & 4.519 & 8.800 & \\
\hline Le Quéré & & & & 8.825 & 16.52 \\
\hline Hortmann, Peric, and Scheure & & 2.245 & 4.521 & 8.825 & \\
\hline Saitoh and Hirosh & & 2.242 & & 8.712 & \\
\hline Ball and Kuo & 1.118 & 2.248 & 4.528 & 8.824 & 16.52 \\
\hline Ho and Lin & 1.118 & 2.248 & 4.528 & 8.824 & 16.52 \\
\hline Comini, Cortella, and Manzan & & & 4.503 & 8.825 & 16.53 \\
\hline Kalita, Dalal, and Dass & 1.118 & 2.245 & 4.522 & 8.829 & 16.52 \\
\hline Present work & 1.118 & 2.242 & 4.528 & 8.822 & 16.80 \\
\hline
\end{tabular}

\subsection{Lid-Driven Cavity Flow Problem}

The lid-driven cavity problem schematic in Figure 10 is investigated in a square with $101 \times 101$ and $129 \times 129$ meshes. At $\mathrm{Re}=5,000$, the predicted velocity profiles $u(0.5, y)$ and $v(x, 0.5)$ in Figure 11 are compared with the steady-state benchmark solutions of Ghia [23]. As can be seen from the extremely good agreement between the simulated and benchmark solutions, the applicability of the proposed scheme to 
high Reynolds number flow simulations is confirmed. For the sake of completeness, the centers of three eddies at T, BL, and BR, schematic in Figure 10, are summarized in Table 1 for $\mathrm{Re}=400,1,000,3,200$, and 5,000. Good agreement with the comparison data [23] is confirmed.

\subsection{Backward-Facing-Step Problem}

The backward-facing-step problem schematic in Figure 12 is investigated at $\mathrm{Re}=800$ with $L$ chosen as the characteristic length. The step geometry chosen for the current study has a step height of $1 / 2$, a channel height of 1 , and the length is 20. The simulated solution plotted in Figure 13 in the uniform mesh $201 \times 41$ is compared with other results tabulated in Table 2 [24]. In Figure 14, comparison with the benchmark solution of Gartling [25] is also made at $x=7$. With good agreement in the comparison studies, the proposed method is verified again.

\subsection{Natural-Convection Problem}

The natural-convection problem schematic in Figure 15 is considered at $\mathrm{Ra}=10^{3}, 10^{4}, 10^{5}, 10^{6}, 10^{7}$, which are calculated with $L$ as the characteristic length, and $\operatorname{Pr}=0.71$. Uniform mesh $81 \times 81$ is employed for the case with $\mathrm{Ra}=10^{3}, 10^{4}$, and $129 \times 129$ for the cases with $\mathrm{Ra}=10^{5}, 10^{6}, 10^{7}$. The simulated solutions plotted in Figures 16-19 compare well with other simulated solutions given in [26] and are tabulated in Tables 3, 4, and 5 .

\section{CONCLUDING REMARKS}

The two-dimensional dispersion-relation-preserving convection scheme is presented to solve the unsteady convection-diffusion transport equation. Both dispersive and dissipative natures of the developed DRP model have been explored. To validate the proposed scheme, we have considered problems amenable to exact solutions. The computed $L_{2}$-error norms and their resulting rates of convergence demonstrate the advantage of employing the proposed scheme to solve for problems having smooth as well as sharply varying solution profiles. A high Reynolds number lid-driven cavity flow problem, a backward-facing-step flow problem, and a naturalconvection problem in a square are also studied. All the simulated solutions are shown to have good agreement with the benchmark solutions.

\section{REFERENCES}

1. D. B. Spalding, A Novel Finite Difference Formulation for Differential Expressions Involving Both First and Second Derivatives, Int. J. Numer. Meth. Eng., vol. 4, pp. 551-559, 1972.

2. B. P. Leonard, A Stable and Accurate Convective Modelling Procedure Based on Quadratic Upstream Interpolation, Comput. Meth. Appl. Mech. Eng., vol. 19, pp. 59-98, 1979.

3. C. R. Swaminathan and V. R. Voller, Streamline Upwind Scheme for Control-Volume Finite Elements, Part I. Formulations, Numer. Heat Transfer B, vol. 22, no. 1, pp. 95-107, 1992. 
4. G. de Felice, F. M. Denaro, and C. Meola, Multidimensional Single-Step Vector Upwind Schemes for Highly Convective Transport Problems, Numer. Heat Transfer B, vol. 23, no. 4, pp. 425-460, 1993.

5. Y. Li and M. Rudman, Assessment of Higher-Order Upwind Schemes Incorporating FCT for Convection-Dominated Problems, Numer. Heat Transfer B, vol. 27, no. 1, pp. 1-21, 1995.

6. T. W. H. Sheu, S. F. Tsai, and M. M. T. Wang, Monotone Multidimensional Upwind Finite Element Method for Advection-Diffusion Problems, Numer. Heat Transfer B, vol. 29, no. 3, pp. 325-344, 1996.

7. T. W. H. Sheu, S. F. Tsai, and S. K. Wang, Monotonic, Multidimensional Flux Discretization Scheme for All Peclet Numbers, Numer. Heat Transfer B, vol. 31, no. 4, pp. 441-457, 1997.

8. C. K. W. Tam and J. C. Webb, Dispersion-Relation-Preserving Finite Difference Schemes for Computational Acoustics, J. Comput. Phys., vol. 107, pp. 262-281, 1993.

9. I. A. Abalakin, A. V. Alexandrov, V. G. Bobkov, and T. K. Kozubskaya, High Accuracy Methods and Software Development in Computational Aeroacoustics, J. Comput. Meth. Sci. Eng., vol. 2, no. 3, pp. 1-14, 2003.

10. F. Q. Hu, M. Y. Hussaini, and J. L. Manthey, Low-Dissipation and Low-Dispersion Runge-Kutta Schemes for Computational Acoustics, J. Comput. Phys., vol. 124, pp. 177-191, 1996.

11. D. W. Zingg, Comparison of High-Accuracy Finite-Difference Methods for Linear Wave Propagation, SIAM J. Sci. Comput., vol. 22, no. 2, pp. 476-502, 2000.

12. M. M. T. Wang and T. W. H. Sheu, An Element-by-Element BICGSTAB Iterative Method for Three-Dimensional Steady Navier-Stokes Equations, J. Comput. Appl. Math., vol. 79, pp. 147-165, 1997.

13. P. M. Gresho and R. L. Sani, On Pressure Boundary Conditions for the Incompressible Navier-Stokes Equations, Int. J. Numer. Meth. Fluids, vol. 7, pp. 1111-1145, 1987.

14. L. Quartapelle and M. Napolitano, Integral Conditions for the Pressure in the Computation of Incompressible Viscous Flows, J. Comput. Phys., vol. 62, pp. 340-348, 1986.

15. T. W. H. Sheu and R. K. Lin, An Incompressible Navier-Stokes Model Implemented on Nonstaggered Grids, Numer. Heat Transfer B, vol. 44, no. 3, pp. 277-294, 2003.

16. H. Johnston and J.-G. Liu, Accurate, Stable and Efficient Navier-Stokes Solvers Based on Explicit Treatment of the Pressure Term, J. Comput. Phys., vol. 199, pp. 221-259, 2004.

17. C. Bogry and C. Bailly, A Family of Low Dispersive and Low Dissipative Explicit Schemes for Flow and Noise Computations, J. Comput. Phys., vol. 194, pp. 194-214, 2004.

18. T. K. Sengupta, G. Ganeriwal, and S. De, Analysis of Central and Upwind Compact Schemes, J. Comput. Phys., vol. 192, pp. 677-694, 2003.

19. J. von Neumann and R. D. Richtmyer, A Method for the Numerical Calculation on Hydrodynamic Shock, J. Appl. Phys., vol. 21, pp. 232-237, 1950.

20. E. Burman and P. Hansbo, Edge Stabilization for Galerkin Approximations of Convection-Diffusion-Reaction Problems, Comput. Meth. Appl. Mech. Eng., vol. 193, pp. 1437-1453, 2004.

21. D. F. Griffiths and A. R. Mitchell, in T. J. R. Hughes (ed.), On Generating Upwind Finite Elements, Finite Element for Convection Dominated Flows, pp. 91-104, AMD, ASME, New York, vol. 34, 1979.

22. R. M. Smith and A. G. Hutton, The Numerical Treatment of Convection-A Performance Comparison of Current Methods, Int. J. Numer. Meth. Heat Transfer, vol. 5, pp. 439-461, 1982.

23. U. Ghia, K. N. Ghia, and C. T. Shin, High-Re Solutions for Incompressible Flow Using the Navier-Stokes Equations and a Multigrid Method, J. Comput. Phys., vol. 48, pp. 387$411,1982$. 
24. I. E. Barton, The Entrance Effect of Laminar Flow over a Backward-Facing Step Geometry, Int. J. Numer. Meth. Fluids, vol. 25, pp. 633-644, 1997.

25. U. D. K. Gartling, A Test Problem for Outflow Boundary Conditions-Flow over a Backward-Facing Step, Int. J. Numer. Meth. Fluids, vol. 11, pp. 953-967, 1990.

26. J. C. Kalita, D. C. Dalal, and A. K. Dass, Fully Compact Higher-Order Computation of Steady-State Natural Convection in a Square Cavity, Phys. Rev. E, vol. 64, 066703, pp. 1-13, 2001. 